\title{
Comparisons between computer simulations of room acoustical parameters and those
} measured in concert halls

Rindel, Jens Holger; Shiokawa, Hiroyoshi; Christensen, Claus Lynge; Gade, Anders Christian

Published in:

Acoustical Society of America. Journal

Link to article, DOI:

$10.1121 / 1.425555$

Publication date:

1999

Document Version

Publisher's PDF, also known as Version of record

Link back to DTU Orbit

Citation (APA):

Rindel, J. H., Shiokawa, H., Christensen, C. L., \& Gade, A. C. (1999). Comparisons between computer simulations of room acoustical parameters and those measured in concert halls. Acoustical Society of America. Journal, 105(2), 1173-1173. https://doi.org/10.1121/1.425555

\section{General rights}

Copyright and moral rights for the publications made accessible in the public portal are retained by the authors and/or other copyright owners and it is a condition of accessing publications that users recognise and abide by the legal requirements associated with these rights.

- Users may download and print one copy of any publication from the public portal for the purpose of private study or research.

- You may not further distribute the material or use it for any profit-making activity or commercial gain

- You may freely distribute the URL identifying the publication in the public portal 


\title{
Posters from various technical sessions remain on display in the Poster Gallery.
}

\section{Session 3pAAa}

\section{Architectural Acoustics and Signal Processing in Acoustics: Verification of Auralization and Modeling Programs I}

\author{
Michael Vorlaender, Cochair \\ Institut für Technische Akustik, Aachen University, D-52056 Aachen, Germany \\ Robert C. Coffeen, Cochair \\ Architectural Engineering, University of Kansas, Lawrence, Kansas 66045, USA
}

Chair's Introduction—4:15

Invited Papers

4:20

3pAAa1. Verification of prediction based on randomized tail-corrected cone-tracing and array modeling. Bengt-Inge L. Dalenbäck (CATT, Mariagatan 16A, SE-41471 Gothenburg, Sweden)

A new room acoustics prediction method, randomized tail-corrected cone-tracing (RTC), is described together with sample prediction/measurement comparisons. RTC is the result of ten years of experience using the image source model, ray- and conetracing, and combines elements of all three methods. RTC is a robust number-prediction method, including the effects of frequencydependent diffuse reflection for both early and late reflections, as well as a method that creates echograms suitable for binaural post-processing and auralization. Further, a WIN32 DLL directivity interface (DDI) is described. The interface is general and is capable of arbitrary data-format translation and accurate distance-dependent high-resolution array modeling including beam steering. The array modeling is either made via a generic DDI module or via modules custom designed based on detailed manufacturer array specifications (transducer placement, directivity pattern, aim, weighting filter, and delay). Sample prediction/measurements comparisons are given for DSP-controlled column arrays under free-field conditions, in a large railway station and in a large church. It is demonstrated that the common fixed measurement distance 10-deg full space data is insufficient for modeling arrays both due to the limited angular resolution and because far-field conditions may not be reached until $30 \mathrm{~m}$ distance or beyond.

4:40

3pAAa2. Report on the second International Round Robin on Room Acoustical Computer Simulation. Ingolf Bork (Projekt 1.401 Physikalisch-Technische Bundesanstalt, D-38116 Braunschweig, Germany)

A Swedish concert hall with a volume of about 11000 cubic meters served as a test object to compare the performance properties of room simulation software programs. In six octave bands and at 12 source-receiver combinations, nine acoustic parameters defined in ISO 3382 had to be calculated. In the first phase of this round robin only the geometry and a written description of the acoustically relevant properties were given, while in the second phase absorption and diffusion coefficients of the surfaces were prescribed for each octave band. The results obtained by the 16 participants from nine countries will be discussed and compared with measurements performed in the hall. The analysis of the discrepancies and the high variance of the calculated data show that the influence of the software user cannot be neglected and a high degree of practical experience in room acoustics is required to produce optimal results.

5:00

3pAAa3. Comparisons between computer simulations of room acoustical parameters and those measured in concert halls. Jens Holger Rindel (Norwegian Inst. of Bldg. Res., P.O. Box 123 Blindern, N-0314 Oslo, Norway), Hiroyoshi Shiokawa, Claus Lynge Christensen, and Anders Christian Gade (Tech. Univ. of Denmark, DK-2800 Lyngby, Denmark)

A number of European concert halls were surveyed in 1989. In this paper comparisons are made between measured room acoustical parameters and those obtained from computer simulations on concert halls using the ODEON program version 3.1. The key parameter compared with measured data is the reverberation time, and this is mainly used to adjust the absorption data of the surfaces in the computer model. But five additional parameters are calculated and compared with measured data as well. In order to determine the sensitivity of the computer model, comparisons are also made between the results obtained from computer simulations using models with a high geometrical fidelity and those from models with simplifications to geometry that are in better agreement with the laws of high-frequency modeling. 
3pAAa4. Real time auralization with EASE 3.0. Wolfgang Ahnert (ADA Acoust. Design Ahnert, Gustav-Meyer-Allee 25, D-13355 Berlin, Germany, wahnert@ada-acousticdesign.de)

The new EASE 3.0 for Windows combines advanced features of the DOS-based system EASE 2.1 with the known ones of the Auralization package EARS 1.1. In case of the DOS-based system, one has to use additional hardware to convolve the calculated impulse responses with the head-related transfer functions and eventually with the dry tests signal. After calculation of the corresponding impulse response one can listen now in EASE 3.0 to the convolved binaural signal by use of just a normal sound card. Additionally, with a duplex sound card one can enter CD signals directly and, after a transient time of $50 \mathrm{~ms}$ to $2 \mathrm{~s}$ (depending on the computer parameters and the sample rate of the signal), one can obtain the auralized results. Another option allows one to calculate the direct sound sequence of used speaker signals in realtime also and then to use the auralization routine to check echo occurrence or correct localization of sound sources. The convolution algorithm used in EASE 3.0 is patent pending and was created in cooperation with LAKE/Australia. In a project still under construction, the measured results have been compared with auralization results obtained by physical scale model measurements. Both results will be demonstrated.

$5: 40$

3pAAa5. Comparison of simulation and measured sound system performance in the German Reichstag Debate Hall, Berlin. Volker Loewer (IFB Rhein Main, Eleonorenstr. 11, D-65474 Bischofsheim, Germany, ifbrm@aol.com)

For the design of the new sound system for the Reichstag Debate Hall, Berlin, the ULYSSES computer aided acoustic design program has been used. The 3-D model of the room consisted of more than 1000 faces treated with different absorption in octave bands according to the architect's plans. Simulation of direct sound coverage, RT60, intelligibility, energy decay via ray-tracing, etc., was done to evaluate different solutions of the sound system designs and their interaction with room acoustics. It is intended to compare actual acoustic test results to simulated predictions and to discuss conformity and deviations. The software was also used to predict directivity balloons of the complex speaker arrays located in the debate hall. The balloons were derived from measured balloons of the single devices used in the arrays. Considering relative mechanical locations, acoustic centers, acoustic power, and delays, the predictions showed good conformity with polar measurements of a real cluster.

6:00

3pAAa6. A computer auralization and "real world" comparison using the computer programs EASE and EARS. Robert C. Coffeen (Architectural Eng., Marvin Hall, Univ. of Kansas, Lawrence, KS 66045) and Gabriel Caunt (Russ Berger Design Group, Inc., Dallas, TX 75244)

In order to compare a "real world" acoustic environment with a computer auralization of the same space, an Episcopal Church located in Prairie Village, Kansas (near Kansas City, Missouri) was selected as the "real" space. Anechoic speech and music recordings were reproduced in this space and recorded binaurally. The same anechoic recordings were introduced into a computer model of this room with auralizations produced by the computer programs EASE and EARS. The "real world" binaural recordings are compared with the computer-generated audio using both objective and subjective methods. The measured impulse response of the room and the computer-predicted impulse response are compared. Comparisons are also made between the measured and the predicted reverberation periods of the room.

WEDNESDAY AFTERNOON, 17 MARCH 1999

ROOM H1028, 4:35 TO 6:20 P.M.

\title{
Session 3pAAb
}

\section{Architectural Acoustics: Sound Insulation and Subjective Annoyance}

\author{
Jens Holger Rindel, Cochair \\ Norwegian Institute of Building Research, P.O. Box 123, Blindern, N-0314 Oslo, Norway \\ Angelo J. Campanella, Cochair \\ Campanella Associates, 3201 Ridgewood Drive, Columbus, Ohio 43026, USA \\ Chair's Introduction—4:35 \\ Invited Papers \\ 4:40
}

3pAAb1. On the utility of extending the low-frequency range of standards for sound isolation in buildings. Stephen J. Lind, Karl S. Pearsons, and Sanford A. Fidell (BBN Technol., 21128 Vanowen St., Canoga Park, CA 91303, slind@bbn.com)

Frequencies above $100 \mathrm{~Hz}$ are commonly considered in sound insulation computations as specified by ASTM E413 and ISO 717. This is appropriate for concerns about speech privacy in interior spaces. However, aircraft ground operations (including takeoff roll, engine run-ups, and thrust reverser deployment) may expose buildings near runways to appreciable amounts of energy at yet lower 
frequencies. Several studies of low-frequency aircraft noise levels have recently been completed in the United States, and the U.S. Federal Aviation Administration is paying for architectural treatments to reduce low-frequency aircraft noise in residences near one airport. This paper describes the findings of a recent social survey of residential annoyance caused by low-frequency runway sideline noise, including noise in the 25 to $80 \mathrm{~Hz}$ one-third octave bands, and reviews other evidence about the role of low-frequency energy in sound isolation metrics.

5:00

3pAAb2. On subjective impact sound insulation classes. Per Hammer (Dept. of Eng. Acoust., Lund Univ., Box 118, 22100 Lund, Sweden, per.hammer@kstr.lth.se) and Erling Nilsson (Lund Univ., 22100 Lund, Sweden)

The possibility to divide impact sound annoyance into classes is investigated. The experiments, based on human perception, are done in a laboratory environment where various floor structures have been included to produce impact noise. These tests are correlated to studies pertained to real-life situations for tenants. The statistical tool to test the significant differences between the classes is Rou-Kupper. Real footsteps are used as a noise source. In addition, airborne sound insulation is treated in a similar manner, whereby one may conclude that there are some difficulties finding classes with statistical significant differences. [Work supported by the Swedish Council of Building Research.]

\section{Contributed Papers}

\section{$5: 20$}

3pAAb3. Group subjective ratings of airborne sound insulation. John Bradley (IRC, Natl. Res. Council, Montreal Rd., Ottawa, ON K1A 0R6, Canada, john.bradley@nrc.ca)

This paper will compare various sound insulation measures as predictors of the grouped subjective ratings obtained from a field survey of 600 subjects and the airborne sound insulation of their 300 party walls. The subjects lived in both row housing and multi-floor apartment buildings in three different Canadian cities. The questionnaire first asked about general issues concerning the subjects building followed by more specific questions rating the sound insulation and the audibility of various noises. Sound transmission loss measurements were made in $1 / 3$ octave bands from 100 to $4000 \mathrm{~Hz}$. In addition to the standard ISO and ASTM single number ratings, 20 different single number sound isolation measures were calculated. Most were simple variations of the standard quantities. The standard STC rating (including the 8-dB rule) was one of the better predictors of most responses. Excluding the 8-dB rule decreased correlations with responses and especially those concerning hearing musical sounds. Several responses were best correlated with transmission loss values in the $160-$ and $200-\mathrm{Hz}$ bands.

\section{5:40}

3pAAb4. Considerations about the influence of traffic noise in the design of new hospitals. Eduardo Bauzer Medeiros (Dept. de Engenharia Mecânica da UFMG, Av. Antônio Carlos 6627, 31270-901, Belo Horizonte-MG, Brazil, flugzbau@dedalus.lcc.ufmg.br) and Ivan de Azevedo Camelier (UBI, 6200, Covilhã, Portugal)

The main origin of noise disturbance in new hospitals located inside densely populated areas can usually be associated with vehicular traffic. However, the effect of this disturbance is often not fully appreciated. Compliance with standard legislation limits is often considered to be a satisfactory design criterion. In fact, even moderately higher noise levels can introduce physiological changes, which may have a decisive influence on the convalescence of a hospital patient. The present work associates some of the information which is available on the effects of patients subjected to different noise levels with considerations about hospital and the associated access roads design. Field measurements carried out in the vicinity of typical existing and planned hospitals are also described and associated with the local traffic conditions. Finally, a few practical guidelines are laid, considering a rest situation of a hospital under construction near an important access road. The objective is to establish a set of acoustic design guidelines for hospitals and the surrounding areas, particularly the road system, aiming at a more efficient convalescence period for the patients.

\section{6:00}

3pAAb5. Vibrations caused by construction work-Measurements and surveillance. Wolfgang Teuber and Ernst-Jo Voelker (Inst. for Acoust. and Bldg. Phys., Oberursel, Germany)

Numerous construction principles applied especially in underground engineering are the sources of vibrations. The measurement of vibrations and evaluation of the results are made based upon different criteria. These criteria are formed taking into consideration the influences upon people in the surrounding area, the risk of damage to buildings and the possibility of the malfunctioning of technical systems such as computers or laser equipment. Measurements were taken while several different construction methods were being applied. Short-term measurements are, for the most part, insufficient and must be completed by long-term research. Maximum acceptable values are defined in existing standards based on the influences of vibrations upon persons and upon various kinds of buildings. For technical equipment, stricter limits had to be defined. When the type and location of construction work changes, the application of an automatic monitoring system is advantageous. Such a system is described which was installed to survey computers with special hard disc drives. Existing measurement results allow for predictions and calculations to be made in order to determine the best method of construction. Some principles of building methods, measurements, and vibration monitoring are mentioned. 


\title{
Session 3pAO
}

\section{Acoustical Oceanography and Underwater Acoustics: Acoustic Tomography and Thermometry II}

\author{
James F. Lynch, Chair \\ Woods Hole Oceanographic Institute, Bigelow 203, Woods Hole, Massachusetts 02543, USA
}

\section{Invited Papers}

$$
\text { 4:20 }
$$

3pA01. Shallow-water tomography: Acoustic maps of the New England shelfbreak front. Ching-Sang Chiu (Dept. of Oceanogr., Naval Postgrad. School, Monterey, CA 93943), James F. Lynch, and Glen Gawarkiewicz (Woods Hole Oceanogr. Inst., Woods Hole, MA 02543)

In the summer of 1996, an integrated acoustic-oceanographic experiment was carried out in the Middle Atlantic Bight to study the dynamics of the shelfbreak front and the effects of the frontal variability on sound propagation. The experiment employed a suite of acoustic and oceanographic sensors, including several sources and two vertical hydrophone arrays (VLAs) straddling the shelfbreak front and a SeaSoar that provided several high-resolution, three-dimensional surveys of the frontal region. In this presentation, the results of an acoustic tomographic analysis of the frontal variability are reported. In deriving the tomographic maps, an adaptive beamformer was first used to detect, resolve, and track individual acoustic ray and modal arrivals from a 224- and a 400-Hz source on the slope to a vertical array on the shelf. A hybrid ray-mode inverse technique was then applied to the resolved arrivals to produce a time series of cross-frontal images of ocean temperature. The tomographic observations are interpreted together with the SeaSoar, ADCP, and thermistor data. [Work supported by ONR.]

\section{4:40}

3pA02. Broadband geo-acoustic inversion in shallow water. Jean-Pierre Hermand (Environ. Res. Div. SACLANT Undersea Res. Ctr., viale San Bartolomeo 400, 19138 La Spezia, Italy, hermand@saclantc.nato.int)

The paper discusses an inversion method that allows the rapid determination of in situ geo-acoustic properties of the ocean bottom without resorting to large acoustic receiving apertures, synthetic or real. The method is based on broadband, waterborne measurements and modeling of the waveguide impulse response between a controlled source and a single hydrophone. Results from 1994-1997 experiments in Mediterranean shallow waters with single elements of vertical array and advanced drifting acoustic buoys are reviewed. Inversion of the bottom parameters is performed with an objective function that includes the processing gain of a model-based matched filter (MBMF) receiver relative to the conventional matched filter. The MBMF reference signals incorporate waveguide Green's functions for the known geometry and water column acoustic model, and hypothesized bottom geo-acoustic models. The inversion results demonstrated that—even for the fairly complex environmental conditions of the experiments—a single transmission of a broadband coded signal received at a single depth and a few hundred of forward modeling runs were sufficient to resolve the bottom features. These included the sound-speed profile, attenuation, density, and thickness of the top sediment layer, and sound speed and attenuation of the bottom. Exhaustive parameter search proved unequivocally the low-ambiguity and high-resolution properties of the MBMF-derived objective. The single-hydrophone bottom inverses closely agree with the ones obtained under the exact same conditions from matched-field processing of multitone pressure fields sampled on a vertical array. Both of these results agree with expectations from geophysical ground thrust.

\section{Contributed Papers}

\section{5:00}

3pAO3. Results for tomographic geoacoustic inversion on simulated multiarray data. A. Tolstoy (Integrated Performance Decisions, Inc., 8610 Battailles Ct., Annandale, VA 22003, atolstoy@ipdinc.com)

Only recently have broadband multiarray data been collected which are suitable for tomographic inversion for the estimation of shallow-water geoacoustic parameters. In particular, this unique data set was collected in the Haro Strait region off the west coast of Canada by N. R. Chapman and colleagues. These data have now been simulated and studied in preparation for the tomographic inversion of the actual data, a very difficult problem involving hundreds of parameters. Results to be discussed here con- cern these simulated data and recent successes in their inversion for the estimation of such properties as sediment sound-speeds and densities over a region where such parameters vary as a function of range, azimuth, and depth. [Work supported by ONR.] $\mathbf{6}: 20$

3pAO4. Acoustic data preprocessing in the TOMOLAB/OCTOPUS project. Mauuary Didier and Bernard Faure (Laboratoire des images et signaux, INP, Grenoble, St. Martin d'heres, France, Didier.Mauuary@lis.inpg.fr)

This paper presents the acoustic data preprocessing task in the TOMOLAB graphic interface tool. TOMOLAB is a goal of the OCTOPUS project, under the MAST III program of the European Com- 
munity. Data preprocessing consists in transforming raw tomographic data into compressed/correlated peaklike data. It is the first step before time delay estimation, time delay identification, and time delay inversion. The complete data processing must perform matched filtering, multisequence averaging, Doppler/phase compensation, and mooring motion correction. A synthetic view is given of several technical choices that integrate these tasks, jointly or successively. This work is based on internal studies and existing pieces of literature. The Hadamard transform, the Fourier matched filtering, fast Doppler processing, phase estimation/correction algorithm, delay shifting in the time or Fourier domain, data format and sampling are discussed. Efficient data processing has to account for the computing speed, the compression ratio, and achievable accuracy of delay estimation.

$5: 40$

3pAO5. Matched-peak tomography. E. K. Skarsoulis (Inst. of Appl. and Computational Mathematics, FORTH, 71110 Heraklion, Crete, Greece)

A new approach is presented for the inversion of travel-time data in ocean acoustic tomography. Using model relations between sound-speed and arrival-time perturbations about a set of background states, arrival times and associated model errors are calculated on a fine grid of model states discretizing the parameter space. Each model state can explain (identify) a number of observed peaks in a particular reception laying within the uncertainty intervals of the corresponding predicted arrival times. The model states that explain the maximum number of observed peaks are considered as the more likely parametric descriptions of the reception; these model states can be described in terms of mean values and variances providing a statistical answer (matched-peak solution) to the inversion problem. An advantage of the proposed approach is that there is no need for initialization of the inversion procedure, i.e., no need for additional hydrographic information. Furthermore, the matched-peak solution for each reception is independent of the previous reception identification/inversion results. The method was applied to nine-monthlong tomography data from the Thetis- 2 experiment in the western Mediterranean sea. [Work partially supported by EU/MAST.]

\section{6:00}

3pAO6. On the simultaneous estimation of current and sound velocity structure in the ocean, using matched-field processing. Michael Taroudakis (Dept. of Mathematics, Univ. of Crete and Inst. of Appl. and Computational Mathematics, FORTH, P.O. Box 1527, 71110 Heraklion, Crete, Greece, taroud@iacm.forth.gr) and Maria Markaki (Inst. of Appl. and Computational Mathematics, FORTH, 71110 Heraklion, Crete, Greece)

A matched-field processing method for the simultaneous estimation of ocean current velocity structure and sound speed profiles, based on reciprocal transmissions of $\mathrm{cw}$ or broadband acoustic signals between two locations in the water column, is presented and discussed. For each transmission, a single source and a vertical array of hydrophones are used. The current velocity is introduced as an additional unknown in the inverse problem, through the concept of the "effective sound speed," being the sum of the actual sound speed and the projection of the current velocity on a vertical plane, defined by the sound source and the receiver. The work is mainly focused on the study of the effect of variations of the current velocity on the various processors proposed for the matched-field inversions and on the inversion procedure itself, under the assumption that neither the actual position of the source receiver system nor the actual sound velocity structure are known. Using synthetic data, it is demonstrated that the results of inversion for the current velocity are reliable, under some limitations posed by the degree of uncertainty for the other unknown parameters. [Work supported by INTAS-RFBR.]

\section{Contributed Posters}

These posters will be on display in the Poster Gallery from Thursday to Friday, 18-19 March. Authors will be at their posters from 10:00 a.m. to 12:00 noon on Friday, 19 March.

3pAO7. The experimental demonstration of selective mode excitation in a shallow-water environment. James C. Preisig (Dept. of Appl. Ocean Phys. and Eng., Woods Hole Oceanogr. Inst., Woods Hole, MA 02543), John R. Buck (Univ. of Massachusetts, North Dartmouth, MA 02747), Mark Johnson (Woods Hole Oceanogr. Inst., Woods Hole, MA 02543), and Hu Dou (Univ. of Massachusetts, North Dartmouth, MA 02747)

The ability to selectively excite single acoustic modes in a shallowwater environment is demonstrated and analyzed. Using vertical source and receiving arrays and the feedback control techniques based on those in Buck et al. [IEEE J. Oceanic Eng. 22, 281-291 (1997)], acoustic modes $1-3$ were excited sequentially for periods up to $20 \mathrm{~min}$ with a fidelity of approximately $60 \mathrm{~dB}$ for mode 1 to $25 \mathrm{~dB}$ for mode 3 . The filtering of the received signal used in the feedback loop by projection into the subspace spanned by the dominant system modes was found to significantly improve the system's performance. This result will be explained and analyzed. The requirement for phase stabilization of the sampled received signal is also presented. The ability demonstrated here for high fidelity selectively controlled modal content of a transmitted acoustic signal offers potential for new methods for acoustic tomography and for studying underwater acoustic propagation. [Work supported by ONR Ocean Acoustics.]
3pAO8. Model of acoustic monitoring of heat and mass transport in the Fram Strait. Konstantin A. Naugolnykh (Environ. Technol. Lab., Boulder, CO 80303), Ola M. Johannessen (Nansen Environ. and Remote Sensing Ctr., Bergen, Norway), Igor B. Esipov, Oleg B. Ovchinnikov, and Yuri I. Tuzhilkin (N. Andreyev Acoust. Inst., Moscow, 117036 Russia)

A computation model of acoustical monitoring of heat and mass transport in the Fram Strait is discussed. The method relies on the advections of small-scale inhomogeneities across the acoustic path and travel-time variations in the process of signal crossing of the Strait on a number of paths to infer the intervening of fine-scale variability and transverse current. Inhomogeneities of the current produce perturbations in the travel time of the sound, and the current can be sensed by generating a time-lagged cross correlation of the full acoustic field. Both the linear four-element transmission array and four-element receiving array with equally spaced elements were used for calculations. By combining the signals from each transmitter-receiver pair in different ways, a number of different path positions were probed and a profile of transverse current $U(r)$ along the propagation path $r$ was retrieved, whereas the value of the travel-time variation is related with $T$-temperature change. The possibility of estimation of heat $Q \sim \int U(r) T(r) d r$ and mass $M \sim \int U(r) d r$ transport in the cross section is regarded. [Work supported by Environmental and Climate Programme of EC, Project PL970338.] 
3pA09. Internal wave inversions for one year of Pioneer-Hawaii ATOC transmissions. Kevin D. Heaney and the ATOC Group ${ }^{\text {a) }}$ (Sci. Applications Intl. Corp., 11803 Sorrento Valley Rd., San Diego, CA 92121)

The ATOC experiment transmitted $75-\mathrm{Hz}$ signals from Pioneer Seamount off the coast of California to a vertical line array (VLA) off Hawaii. The combination of VLA and long-range propagation provides the opportunity to perform mode-based internal wave tomography. Each arrival is mode-filtered and the statistics of the mode arrivals are tabulated. These individual mode arrival time (centroid) and spread are then compared with calculated mode statistics from broadband PE modeling of the propagation path. In this manner a statistically robust inversion for the internal wave strength is performed. The weakness of the inversion is the sensitivity to the bottom scattering near the source. Results for one year of data will be presented for two different bottom types. The long-scale time series may indicate possible sources of internal waves in the ocean. Small time scale variations may be attributable to internal tides. ${ }^{\text {a) }}$ The ATOC Group: A. B. Baggeroer, D. Menememlis, and C. Wunsch (MIT); T. Birdsall, K. Metzger (Univ. of Michigan); C. Clark (Cornell Univ.); J. Colosi (WHOI); B. D. Corneulle, M. Dzieciuch, W. Munk, P. F. Worcester (SIO); D. Costa (Univ. of California, Santa Cruz, CA); B. D. Dushaw, B. M. Howe, J. A. Mercer, and R. C. Spindel (APL-U Wash.); A. M. G. Forbes (CSIRO, Hobart).

3pA010. AMOC: Preliminary results. Hanne Sagen (NERSC, Edv. Griegsvei 3A, 5037 Solheimsviken, Norway, hanne.sagen@nrsc.no), Ola M. Johannessen, Halvor Hobaek, and Sigve Tjoetta (Univ. of Bergen, Bergen, Norway)

The scope of this presentation is to give preliminary results of the sensitivity study of sound propagation in the Arctic including the Fram Strait using propagation loss observations and existing acoustic propagation models. Special attention to the consequences for design of future acoustic monitoring concepts will be made. One of the important consequences of the preliminary results is the conflict between the optimum transmission frequency and the optimum frequency for retrieving information about the ice cover. If an experiment is designed to propagate over long distances at a relatively low frequency, then the reflection from a "smooth" ice cover is total and there will be no information about the ice thickness or internal properties of the ice cover. The attenuation due to the ice will be dominated by scattering from rough surface (large facets). So this type of experiment will give information about the averaged temperature through the travel times, while the ice information will be limited. Therefore two concepts have to be considered: one to measure ice parameters, which will need signals composed by appropriate frequencies, and another concept to measure averaged temperature, which needs low frequencies insensitive to ice cover.
3pAO11. Method of the main informative components for acoustic tomography. Alexander Ya. Kalyuzhnyi (Inst. of Hydromechanics, 8/4 Zhelyabov Str., Kiev, 252057, Ukraine) and Leonid G. Krasny (Ericsson, Inc., RTP, NC 27709)

Techniques for an ocean acoustic tomography are based on the representation of the sound-speed field (SSF) by an expansion of some orthogonal basis functions. It is usually assumed that the most efficient basis functions for the SSF expansion are so-called empirical orthogonal functions (EOF). Meanwhile, the experiments show that algorithms based on the EOF can be extremely sensitive to slight errors in the assumed signal and noise characteristics. A new improved algorithm for acoustic tomography will be presented which minimizes output statistical errors of the SSF estimation. The concept of this algorithm is to use eigenvectors of the Fisher information operator as the basis functions into the SSF expansion. The algorithm involves a quadratic inequality constrain on the SSF expansion coefficients to select the most informative components among all the eigenvectors. It is shown that it leads to a robust algorithm in which statistical errors do not accumulate. The performance of the proposed algorithm will be illustrated both for deterministic and random signals.

3pAO12. An ocean acoustic tomography experiment in the central Labrador Sea-First results. Detlef Kindler and Uwe Send (Inst. für Meereskunde, Univ. Kiel, Düsternbrooker Weg 20, D-24105 Kiel, Germany)

First results of an ongoing acoustic tomography experiment will be presented. Since August 1996 a mooring array consisting of four tomography transceivers $(400 \mathrm{~Hz})$, redeployed in the summers of 1997 and 1998, has been measuring acoustic travel times over distances of typically 170$300 \mathrm{~km}$ in the central Labrador Sea. The purpose of such measurements is to estimate time series of horizontally integrated properties like ocean heat content and vertical temperature stratification by inverting travel times into sound speeds and hence into heat contents and temperatures. These large-scale properties will be used to study the interannual variability of ocean heat budget and water masses formed in close connection to deep convection and the ensuing renewal of Labrador Sea Water (LSW) [R. A. Clarke and J. C. Gascard, J. Phys. Oceanogr. 13, 1764-1778 (1983)]. The variability during winter results from destabilization of the water column near the ocean surface caused by atmospheric cooling, subsequent mixing down to $1000-2000 \mathrm{~m}$, and the following restratification of the convection area. The investigations are focused on the upper $2000 \mathrm{~m}$ of the water column, which are well insonified by several trackable ray paths with enough acoustic energy to be processed. [Work supported by Deutsche Forschungsgemeinschaft.] 


\title{
Session 3pEA
}

\section{Engineering Acoustics and Underwater Acoustics: Recent Advances in Underwater Transducers II}

\author{
Elizabeth A. McLaughlin, Cochair \\ Naval Undersea Warfare Center, Code 2131, Newport, Rhode Island 02841-1708, USA \\ Didier Boucher, Cochair \\ DCN Ingénierie Centre SUD, Sous-Direction Lutte Sous La Mer, BP 30, 83800 Toulon Naval, France
}

\section{Contributed Papers}

4:00

\begin{abstract}
3pEA1. Application of the Tonpilz design for optimizing parameters of rod transducers. Boris Aronov (Acoust. Res. Lab., Dept. of Elec. and Computer Eng. and Ctr. for Marine Sci. and Tech., Univ. of Massachusetts, Dartmouth, North Dartmouth, MA 02747)
\end{abstract}

The optimum design of a rod transducer under various loading conditions and operating requirements is examined. It is often possible that the operational requirements can be met by using only a part of the rod volume with the electromechanical or piezoelectric element. The remaining sections of the rod can be replaced by passive materials or caps (i.e., a "head" and a "tail") while still maintaining the same input impedances and resonant frequency. Such a composite rod transducer made of active and passive materials having different length and cross-sectional areas is of the "Tonpilz" type. The proper choice of dimensions and materials for the caps will result in optimum transformation of mechanical (acoustical) loads and transducer performance. Both criteria of optimization, the rod transducer parameters and the procedure for determining properties of the caps that provides optimal loads, are discussed.

4:20

3pEA2. Large aperture 1-3 composite longitudinal resonator (tonpilz) transducer. Charles W. Allen, W. Jack Hughes, and David J. Van Tol (Appl. Res. Lab., Penn State Univ., P.O. Box 30, State College, PA 16804)

A large aperture underwater transducer utilizing 1-3 piezoelectric composite material that operates as a longitudinally resonant (tonpilz) device with a resonance frequency of $45 \mathrm{kHz}$ has been fabricated and tested. The transducer consists of continuous plates of steel and aluminum that act as the rear and front masses, respectively, and a motor section of continuous 1-3 PZT-5H composite. The active radiating face (aluminum front mass) has dimensions of 6.3 in. (4.7 wavelengths) and $3.9 \mathrm{in.} \mathrm{(2.9} \mathrm{wavelengths).}$ Conventional transducers or arrays with radiating faces that have dimensions of over one wavelength require dicing or separate elements to reduce the effect of lateral modes in the components. The tonpilz transducer utilizing 1-3 composite material requires no dicing of any of its components, so fabrication of large aperture arrays that only require a single forward-looking narrow beam are greatly simplified. The measured acoustic responses versus frequency and angle demonstrate that there are no extraneous modes that affect the acoustic performance of the transducer and they compare well to theory for a one-dimensional longitudinal resonator. [Work sponsored by the Scripps Institution of Oceanography, University of California, San Diego.]
4:40

3pEA3. The development of a doubly curved acoustic array using injection molded 1-3 piezocomposite. Kim C. Benjamin, Stephen F. Oliver, Jose C. Arteiro (Naval Undersea Warfare Ctr. Div. Newport, 1176 Howell St., Newport, RI 02841, benjaminkc@code20nl.npt.nuwc.navy.mil), Russell S. Petrucci, and William J. Serwatka (Mater. Systems, Inc., Littleton, MA 01460)

The design, fabrication, and acoustic calibration of a doubly curved acoustic array for UUV oceanographic applications is presented. The sensor employs a unique piezoceramic thermoplastic composite which is conformable and capable of deep ocean operation. The composite consists of several small diameter $(1.0 \mathrm{~mm})$ PZT-5H rods regularly distributed in a two-dimensional lattice and aligned perpendicular to their diameter. The PZT-5H rods are encapsulated within a thermoplastic viscoelastic moldable epoxy matrix to form an active acoustic composite panel. The thermoplastic epoxy phase of the composite allows the panel to be shaped to a specific vehicle geometry. Low-profile $(<1.0 \mathrm{~mm})$ coaxial cable embedded within the composite panel provides addressing between the acoustic array elements and the processing electronics. [This work was supported by Code 80 of the Naval Undersea Warfare Center, Division Newport.]

5:00

3pEA4. Ultra-wide bandwidth backscattering measurements using a transparent flat PVDF sheet source. Scot F. Morse (Code 7136, Naval Res. Lab., Washington, DC 20375), Karen Gipson (Univ. of Puget Sound, Tacoma, WA 98416), Greg Kaduchak (Los Alamos Natl. Lab., Los Alamos, NM 87545), Kevin L. Williams (Univ. of Washington, Seattle, WA 98105), Brian T. Hefner, and Philip L. Marston (Washington State Univ., Pullman, WA 99164-2814)

A thin extended flat PVDF film surrounded by water is demonstrated to be suitable for exploring scattering responses of complicated targets over a range of frequencies from below $2 \mathrm{kHz}$ to $1000 \mathrm{kHz}$. The target lies close to the source, and in the configuration normally used, the scattering passes through the source to a distant hydrophone. While initial experiments [Kaduchak et al., J. Acoust. Soc. Am. 97, 2644-2708 (1995)] explored the resonances of spherical shells for which analytical solutions are known, recent experiments have explored novel features in the responses of truncated tilted cylinders that are either hollow [Morse et al., J. Acoust. Soc. Am. 103, 785-794 (1998)] or solid [K. Gipson, Ph.D. thesis, Washington State Univ. (1998)]. The spectrum radiated by the sheet can be altered by using voltage-step, voltage-pulse, or weighted chirp excitations. An equivalent circuit model is useful for describing the radiated spectrum. Crisp tone bursts have also been produced and used to study the lowfrequency resonances of a steel shell in Lake Union (Seattle). [Work supported in part by the Office of Naval Research and by APL.] 
compression strength. Evidence of damage was found at stresses as low as one-third of the compressive strength. [Work supported by ONR and SPAWAR.]

3pEA5. Mechanical properties of lead magnesium niobate/lead titanate ceramics. Elizabeth A. McLaughlin and Lynn Ewart (Naval Undersea Warfare Ctr., Code 2131, 1176 Howell St., Newport, RI 02841)

This research examines the mechanical properties of doped electrostrictive lead magnesium niobate / lead titanate (PMN-PT). While optimization of the electromechanical properties has been the focus of the materials development effort, mounting anecdotal evidence indicates that there are problems with the mechanical integrity of PMN-PT. Mechanical testing has been employed (1) to measure the flexural strength of doped PMN-PT and understand the causes of failure, and (2) to measure the compression strength of PMN-PT and identify the onset of microstructural damage. Navy type-III ceramic (PZT8) was measured as a benchmark. The average flexural strength of poled PZT8 was found to be $110 \mathrm{MPa}$. Average flexural strengths ranging from 44 to $60 \mathrm{MPa}$ were measured for batches of barium- and lanthanum-doped PMN-PT. Fractography identified the causes of failure. Process modifications, by one vendor, have increased the flexural strength by $38 \%$. The average compression strength of unpoled PZT8 was found to be $1040 \mathrm{MPa}$. An average compressive strength of $890 \mathrm{MPa}$ was measured for barium-doped PMN-PT. Prior to failure, a ceramic develops microstructural damage at levels below the

\section{$5: 40$}

3pEA6. Evidence of nonlinear losses in electrostrictive materials. Harold C. Robinson (Naval Undersea Warfare Ctr., Newport, RI 02841)

It has been well established that the equivalent piezoelectric properties used to model the large signal properties of electrostrictive materials such as lead magnesium niobate/lead titanate (PMN/PT) are functions of the operating conditions. Since these materials are nonlinear, DC bias field, AC drive field, prestress level, and temperature all affect the piezoelectric constants and the coupling factor. However, in transducer models based on equivalent circuits, the dielectric loss is represented as a constant under all of these conditions. Moreover, the dielectric loss tangent typically used in these models must either be inferred from transducer data or be measured under conditions (such as using an RLC meter) that do not represent the actual conditions of operation. This paper will present data, from both material and transducer measurements, suggesting that the losses in electrostrictive materials are strong functions of the temperature, drive field, and compressive prestress. Possible approaches to obtaining improved loss measures from material measurements will be discussed.

\title{
Session 3pED
}

\section{Education in Acoustics: Take Fives-Sharing Ideas for Teaching Acoustics}

\author{
Uwe J. Hansen, Cochair \\ Department of Physics, Indiana University, Terre Haute, Indiana 47809, USA \\ Armin Kohlrausch, Cochair \\ IPO Center for Research on User-System Interaction, P.O. Box 513, NL-5600 MB Eindhoven, The Netherlands
}

Do you have a novel demonstration, a new laboratory experiment, a favorite video, a recorded sound example, or a new idea for teaching acoustics which you are willing to share with your colleagues? At this session a sign-up board will be provided for scheduling presentations. No abstracts are printed. Presenters are encouraged to have handouts to distribute. Multiple presentations are acceptable (not consecutively). Presentations are limited to 5 minutes. Keep them short! Keep them fun! 
Session 3pMU

\title{
Musical Acoustics: Musical Instruments and Structural Acoustics II: Piano and Related Instruments
}

\author{
Isao Nakamura, Cochair \\ 1-33-25 Kokuryo, Chofu, Tokyo 182-0022, Japan \\ Ingolf Bork, Cochair \\ Physikalische Technische Bundesanstalt, Bundesallee 100, D-38116 Braunschweig, Germany \\ Chair's Introduction-4:15 \\ Invited Papers \\ 4:20
}

3pMU1. Piano string excitation: The question of gravity influence on the sound spectra. Anatoli Stulov (Dept. of Mech. and Appl. Mathematics, Inst. of Cybernetics, Akadeemia tee 21, Tallinn, EE0026, Estonia)

The influence of gravity on the process of the grand piano hammer-string interaction was appreciated by D. E. Hall [J. Acoust. Soc. Am. 79, 141-147 (1986)]. Using the reasonable physical assumptions, it was shown that the gravity influence is small and may not be taken into account. It is obvious, but it is important to know exactly what this influence is. The vibration spectra of different flexible strings excited by piano hammers in the gravity field and without gravity were analyzed here. Of course, the influence of gravity increases with the increasing of the contact time. Its depends on the hammer velocity; therefore, the string excitation by hammers with velocities less than $0.5 \mathrm{~m} / \mathrm{s}$ was considered. It was shown that: the gravity influence is greater for the smooth hammers, and this influence is more essential for the middle range of notes; for the small velocities of hammers the gravity changes the mode energy levels of the primary harmonics up to $10 \mathrm{~dB}$, if the hammer strikes only one string of the triple set; the gravity must be taken into account definitely, if the contact time is greater than $5 \mathrm{~ms}$.

4:40

3pMU2. Experimental analysis of two-dimensional vibration of a piano string measured with an optical device. Hideyuki Tanaka, Keinosuke Nagai, and Koichi Mizutani (Inst. of Appl. Phys., Univ. of Tsukuba, Tsukuba Ibaraki, 305-8573 Japan)

It is well known that a piano string vibrates in the direction not only perpendicular to the soundboard but also parallel to it. The sound decays rapidly at first and then it decays slowly. A change in the predominant direction of vibration of the string from perpendicular to parallel contributes to the compound decay rate [G. Weinreich, Sci. Am. 240, 118-127 (1979)]. In this paper, two-dimensional vibration of a piano string is measured with handmade equipment consisting of two phototransistors. Just after the string was struck with a hammer, it vibrates perpendicularly to the soundboard. Then it vibrates gradually in the direction parallel to the soundboard. As a result, a point of the string moves on an elliptic trajectory. It was found that the fundamental resonant frequency of the perpendicular vibration of the E1 string, for example, which is about $40 \mathrm{~Hz}$, is $0.07 \mathrm{~Hz}$ lower than that of parallel vibration. This causes the change of rotating direction of the string every $7 \mathrm{~s}$. The discrepancy of the resonant frequencies is also found in the vibration of the $\mathrm{D} \# 1$ string.

\section{5:00}

3pMU3. Damping of string motion, a significant parameter for sound quality. Klaus Wogram (Phys.-Techn. Bundesanstalt, Proj. 1.401, Bundesallee 100, D-38116 Braunschweig, Germany, klaus.wogram@ptb.de)

To improve the sound quality of pianos and upright pianos the piano maker mostly plans a change of the construction of the backs and the soundboard with its ribs, bridges, and back posts. Unfortunately he often disregards that all these efforts cannot be successful if the damping of the strings is insufficient. Its influence on the sound quality can be very much stronger than that of the soundboard. Guided by some examples, the importance of the dampers, their construction, their mass, and their position will be discussed. In addition the reduction of sound quality due to an insufficient damping of the not-speaking part of a string will be demonstrated.

\section{$5: 20$}

3pMU4. The influence of inharmonicity on piano tuning. Taro Mori and Ingolf Bork (Physikalisch-Technische Bundesanstalt, Bundesallee 100, 38116 Braunschweig, Germany, taro.mori@ptb.de)

It is well known that octaves of piano sounds are stretched to more than a $2: 1$ ratio. One reason is that partials of piano tones are not exactly harmonic because of the flexural rigidity of the string. This inharmonicity is characterized by the coefficient $B$, which depends on the tension, length, Young's modulus, the area of cross section, and the radius of gyration of the string. Piano technicians are trained to tune the octave as "beautifully" as possible, but the inharmonicity of the string hinders perfect tuning without beat. For example, when the second partial of the lower sound and the first partial of the upper sound are tuned without beat, all other partial pairs show beats. On the other hand, since the timbre structure of partials is unique for each instrument, the tuned octaves of two different instruments may differ. Some piano sounds were simulated by additive synthesis with different octave, inharmonicity, and timbre in order to perform subjective hearing tests with some piano tuners. It could be shown that the tuned octave width depends not only on the coefficient $B$ but also on the timbre. The influence of the sound board is also discussed. 
3pMU5. Psychoacoustic model for calculating the sound quality of piano tones. Miriam N. Valenzuela (Inst. of Mech., Faculty of Aerosp. Technol., Univ. of the Federal Armed Forces, Munich, D-85577 Neubiberg, Germany, miriam.valenzuela@unibw-muenchen.de)

Assuming that only audible dissimilarities between different piano tones may lead to different quality judgments, experiments were conducted to determine the perceptual attributes listeners use to judge these dissimilarities. The psychoacoustic results showed that two major attributes contribute with over $90 \%$ to the explanation of the perceived dissimilarities: the psychoacoustic "sharpness" and a second attribute that was described with the scale "open-closed." Both attributes depend on the specific loudness distribution of the sound signals and are defined as a weighted first moment of the critical-band rate distribution of specific loudness using different weighting functions. The weighting function used to calculate the second attribute emphasizes spectral components around $1 \mathrm{kHz}$. The model developed for calculating audible dissimilarities between piano tones showed a good agreement between psychoacoustically measured and calculated dissimilarities; the correlation was 0.90 . The influence of the two attributes on sound quality judgments was verified by listening tests with appropriately modified piano sounds. The results showed that calculating the sound quality of musical instruments on the basis of their sound signals requires an aurally adequate signal processing. The model developed to calculate the sound quality of piano tones showed a good agreement between measured and calculated quality orders.

\title{
Contributed Paper
}

\section{6:00}

3pMU6. Stiffness of strings: Significance in musical acoustics. Edgar Lieber $^{\text {a) }}$ (former member of "Institut für Musikinstrumentenbau," D-08267 Zwota, Germany)

In a linear differential equation of a vibrating string there is a link representing stiffness of the string. In nonlinear theory, string stiffness has not yet been taken into consideration. The author has completed this by means of the Lagrange II method and presents essential results. The significance of string stiffness is different for the different groups of instru- ments. Pianos have remarkably stiff strings. This is inevitable and also necessary because stiffness participates essentially in forming vividness of sound. The well-known "stretched octaves", and also the typical tuning curve are caused by this. For plucked strings, stiffness forms character of sound, together with choice of plucking spot on string and with corpus. For bowed strings, it is preferable to have strings, with minor stiffness, because the interaction of bow/string is better. The effects of jitter and rippling (supplement of sound with statistic noise effects) are better formed by less stiff strings. For wound strings, minor stiffness is possible with cores of filament structure. Theory and examples will be discussed.

a) Present address: Erich Ohser Str. 10, D-08525 Plauen, Germany.

WEDNESDAY AFTERNOON, 17 MARCH 1999

ROOM H107, 4:55 TO 6:20 P.M.

\section{Session 3pNSa}

\section{Noise: Tribute to Henning von Gierke}

\author{
Richard L. McKinley, Cochair \\ Armstrong Laboratories, AL/CFBA, 2160 7th Street, Wright-Patterson AFB, Ohio 45433-7901, USA
}

Alice H. Suter, Cochair

Alice Suter and Associates, 575 Dogwood Way, Ashland, Oregon 97520, USA

Chair's Introduction-4:55

\section{Invited Papers}

5:00

3pNSa1. Henning E. von Gierke: Mentor, colleague, and public policy leader. Alice H. Suter (Alice Suter and Assoc., 575 Dogwood Way, Ashland, OR 97520) 
3pNSa3. The major historical events in the relating of noise-induced hearing loss to noise exposure. Daniel L. Johnson (Interactive Acoustics, 4719 Mile High Dr., Provo, UT 84604)

One event was the recognition of "boilermaker's disease." Another was some of the threshold shift studies during and after WWII that led to the BENOX report. After these developments, there were a series of key events and decisions that have led up to our current state of knowledge and procedures. How Henning von Gierke was influential in most of these and how his decisions have consistently proven to be correct will be demonstrated. For example, he contributed to the 1953 BENOX report, promoted the "equal energy concept," conceived and patented the first noise dosimeter, oversaw the EPA "levels" and criteria documents, and was the chair of the working group that wrote the current international standard (ISO-R1999) that relates noise exposure to noise-induced hearing loss. He was also instrumental in supporting many key research issues such as fetal noise exposure, asymptotic temporary threshold shift, and the longitudinal hearing of children. Henning's perseverance and dedication has provided society with an answer to one of his favorite questions, "how much noise is too much?"

6:00

3pNSa4. 50-year legacy of Henning E. von Gierke's scientific and engineering excellence. Richard L. McKinley (Armstrong Laboratories, AL/CFBA, 2160 7th Street, Wright-Patterson AFB, OH 45433-7901)

WEDNESDAY AFTERNOON, 17 MARCH 1999

ROOM H2032, 4:20 TO 6:20 P.M.

\title{
Session 3pNSb
}

\section{Noise: Rotor Noise from Helicoptors and Propellers}

\author{
Werner Dobrzynski, Cochair \\ DRL-Instiitut für Entwurfsaerodynamik, Lilienthalplatz 7, 38108 Braunschweig, Germany \\ Feri Farassat, Cochair \\ NASA Langley Research Center, Hampton, Virginia 23681-0001, USA
}

Invited Papers

3pNSb1. European helicopter rotor noise research-Highlights of recent projects. Wolf R. Splettstoesser (Deutsches Zentrum für Luft-und Raumfahrt e. V., Institut für Entwurfsaerodynamic, Lilienthal-platz 7, 38108 Branschweig, Germany, wolf.splettstoesser@dlr.de)

Significant advances in understanding, modeling, and controlling helicopter rotor noise have been made in the course of several major European rotor aeroacoustic research projects over the past years. The availability of the German-Dutch Wind Tunnel (DNW), in particular, has enabled research on relatively large main rotor models (up to typically $40 \%$ full scale). Here, the simultaneous application and use of blade-mounted absolute pressure sensors to study the very aeroacoustic source mechanisms, of advanced acoustic-data acquisition systems for the radiated noise, of non-intrusive flow visualization as well as flow and blade deflection measurement techniques to investigate the rotor wake and the blade dynamic response has provided much insight into the physics of rotor aeroacoustics, particularly into the rotor blade-vortex interaction (BVI) impulsive noise phenomenon. In efforts towards controlling BVI impulsive noise, in some research projects, active blade-root control techniques have been investigated to favorably affect the BVI geometry and thus minimize the impulsive noise radiation. Much of the experimental data acquired served as benchmark information to enhance the mathematical modeling of the rotor wake, the rotor airloads, and the noise radiation. In this lecture, several major European helicopter aeroacoustic research projects are discussed. 
3pNSb2. Analysis and capability of a noncompact integration method for rotor noise prediction. J. Prieur (ONERA/Dept. of Numerical Simulation and Aeroacoustics, 29 avenue de la division Leclerc, 92322 Chatillon Cedex, France)

Rotor noise computations starting from CFD input data using either the Kirchhoff or the generalized Ffowcs Williams-Hawkings surface integral methods in unsteady conditions may be very lengthy. This is the case for directivity studies or noise level contour calculations for which a high number of observer locations is required. To overcome this drawback, a fast integration method has been designed. It starts from the emission time which avoids having to solve the retarded time equation and uses directly as integration grid the CFD rotating grid (a priori not adapted to acoustic calculations). This is made possible thanks to a noncompact treatment of each grid element. The method has already been successfully applied in a transonic rotor case using indifferently a subsonically or supersonically rotating CFD grid for the surface integration (G. Rahier and J. Prieur, 53rd Forum of the American Helicopter Society, 29 April-1 May 1997). The method is made more understandable based on a new and clearer justification and its possibilities of extension are discussed.

\section{5:00}

3pNSb3. Analysis of the excess noise of aircraft pusher propellers. Johan B. H. M. Schulten (Aeroacoustics Dept., Natl. Aerosp. Lab. NLR, P.O. Box 153, 8300 AD Emmeloord, The Netherlands, schulten@nlr.nl)

A pusher propeller configuration offers several advantages over the traditional tractor arrangement. For instance, the wing of a twin-engined aircraft is no longer exposed to the swirling and turbulent slipstream. This results in a much smoother wing inflow, a prerequisite for aerodynamic refinement. Acoustically, the more aft position of the propellers considerably reduces the noise impact on the fuselage, yielding the potential of a low cabin noise level. However, in general, the exterior noise of pusher propellers is augmented by the interaction with impinging wakes and exhaust jets. The present paper addresses the physical mechanism of this interaction by applying an unsteady lifting surface theory to the propeller blades. This requires the incident field to be resolved in circumferentially harmonic Fourier components. For each component the unsteady blade pressure distribution is computed as source input for an acoustic calculation. From sample computations for typical operating conditions it appears that the interaction substantially contributes to the total radiated noise of pusher propellers. The present method offers the possibility to investigate in detail the effect of alterations in blade design and flight conditions on tonal level and directivity.

3pNSb4. Recent developments in helicopter rotor noise prediction at NASA. Kenneth S. Brentner (NASA Langley Res. Ctr., Hampton, VA 23681-0001, k.s.brentner@larc.nasa.gov)

Significant progress has been made recently toward the efficient and robust prediction of helicopter high-speed impulsive noise. The far-field approximation to the Ffowcs Williams-Hawkings (FW-H) quadrupole has been augmented with a rigorous mathematical development. Two new quadrupole formulations-a retarded-time formulation known as Q1A and an emission-surface formulation known as Q2-have been developed and implemented for subsonic and supersonic source motion, respectively. A key numerical advantage of the far-field approximation is that volume integration of the source region surrounding the rotor blades is only performed once in a preprocessing stage. The resulting quadrupole noise computation is reduced to surface integration over the rotor disk in the vicinity of the blades. The solution-adaptive marching-cubes integration algorithm used for the emission-surface formulation will be described. Another recent advance in helicopter noise prediction is the use of the FW-H equation on a permeable integration surface surrounding the source region. This application of the $\mathrm{FW}-\mathrm{H}$ equation has been shown to be both analytically and numerically superior to the Kirchhoff approach. Furthermore, numerical evidence is presented to demonstrate that the Kirchhoff method is inappropriate when a wake convects through the surface.

\section{Contributed Papers}

\section{5:40}

3pNSb5. Passive-active low-frequency vibroacoustical fault diagnosis method. Nadejda I. Bouraou (Dept. of Orientation and Navigation Systems, Natl. Tech. Univ. of Ukraine, 37 Peremogy pr., Kiev, 252056, Ukraine, nadya@burau.inec.kiev.ua), Leonid M. Gelman, and Pavel I. Marchuk (Natl. Tech. Univ. of Ukraine, Kiev, 252056, Ukraine)

The presented work is dedicated to the development of the new passive-active low-frequency automatic vibroacoustical early fault diagnosis method of helicopter engine units (compressor stages, turbine stages). The proposed method is based on signal processing of the rotating details of acoustic noise in the low-frequency range $(0-25 \mathrm{kHz})$. There are two important characteristics of this method: (a) it uses a nonstationary narrow-band vibration excitation with variable central frequency, and (b) this excitation is provided by rotating rotor, and user of the method will vary the central frequency, and (b) this excitation is provided by rotating rotor, and user of the method will vary the central frequency of rotor rotation. The basis for using this method is the fact that, at the presence of fault (for example, fatigue crack) in testing details, parameters of arising free and forced oscillations change (nonzero spectral amplitudes of higher harmonics). The new analytical dependencies of testing object oscillation parameters from the relative crack size and parameters of nonstationary excitation are received. A multidimensional testing data vector for diagnostics and evaluation of faults is proposed and investigated.
3pNSb6. An advanced field measurement system for rotorcraft flight noise. James C. Yu and Kenneth D. Wright (NASA LaRC, MS 235, Hampton, VA 23681-0001)

A Langley-developed field acoustic measurement system has been extensively employed to support the NASA aircraft and rotorcraft flight noise research programs since the early 1980s. The system was first successfully used in the NASA Forward Flight Effects on Turbofan Noise program. Starting from the late 1980s, driven by the increased demand on expanded measurement capabilities for rotorcraft flight noise, an advanced field measurement system development program was initiated to support the rotorcraft flight noise measurement, reduction, and prediction. The paper reviews the three major stages of this extensive development program: the analog measurement system stage, the digital measurement system stage, and the digital remote acquisition and storage system stage. The data processing technology associated with these different stages of the system will be presented. The paper will discuss the research drivers for the measurement system development, the development strategy used to meet the system requirement, and lessons learned at each stage of the development. Discussion will also be given on the future rotorcraft flight noise measurement challenges and the advanced measurement systems required to meet these challenges. 


\title{
Session 3pPA
}

\section{Physical Acoustics: Duct Acoustics II}

\author{
Walter Eversman, Cochair \\ Mechanical and Aerospace Engineering and Engineering Mechanics, University of Missouri, 202 Mechanical Engineering, \\ Rolla, Missouri 65401, USA \\ Dirk Ronneberger, Cochair \\ Drittes Physikalisches Institut, University of Göttingen, Burgerstrasse 42-44, D-37073 Göttingen, Germany
}

\section{Invited Papers}

3pPA1. Interaction between resonators and fluid motion in ducts. Dirk Ronneberger (Drittes Physikalisches Institut der Universitaet Goettingen, Buergerstrasse 42-44, D-37073 Goettingen, Germany, ro@ physik3.gwdg.de)

Each time one is confronted with unwanted narrow-band noise which propagates along a duct, it suggests itself to make use of resonators in the suppression of this noise. However, resonators may be highly sensitive to superimposed mean flow: Detuning and drastic decay of the quality factor is encountered in many cases, nevertheless, also the contrary may happen, namely the increase of the acoustic energy in the resonator. This may result in self-excited oscillations or in the amplification of the incident sound. The paper gives a review of these phenomena, and it will focus on the physical effects behind these phenomena. Simple models that are based on the governing physical effects have been developed and are compared to experimental results with different configurations of resonators in flow ducts. In the case of sound amplification the amplitude of the organized motion of the fluid may become very high so that the nonlinearity of the interaction with the mean flow becomes significant. The state of knowledge and the open questions in this respect will be addressed as well.

\section{4:40}

3pPA2. Active noise control by use of an aeroacoustic instability. Björn Lange and Dirk Ronneberger (III. Phys. Inst., Bürgerstr. 42-44, D-37073 Göttingen, Germany)

Ducts that are lined with a series of equally spaced cavities exhibit strong amplification of sound waves under specific flow conditions. The amplification is caused by an aeroacoustic instability wave traveling in the boundary layer of the lining. Since the effect is coupled with a drastic variation of the static pressure drop that depends on the amplitude of incident sound, the possibility is implied to efficiently generate high intensity sound at low frequencies: An incident sound wave with harmonically varying amplitude will cause a periodic modulation of the pressure drop and thus lead to the generation of sound at the modulation frequency. Based on these observations, an aeroacoustic loudspeaker has been designed combining a lined duct section as part of a flow channel and a conventional loudspeaker. The output power of the device amounts to up to $95 \mathrm{~dB}$ re: $1 \mathrm{pW} / \mathrm{Hz}$ at $\mathrm{Ma}=0.25$ for frequencies ranging between 0 and $200 \mathrm{~Hz}$ - an observation of some significance with regard to the fact that traditional loudspeakers tend to be rather inefficient at low frequencies. As a feasibility test, the aeroacoustic loudspeaker is used as an actuator in an active noise control setup. High attenuation of low-frequency noise is achieved.

\section{5:00}

3pPA3. Computational aeroacoustics of ducts using parallel computers. Lyle N. Long (Dept. of Aerosp. Eng., Penn State Univ., 233 Hammond Bldg., University Park, PA 16802)

This paper will discuss recent attempts at using computational aeroacoustics (CAA) algorithms on parallel computers to solve duct propagation problems. The algorithms are higher-order accurate methods for solving the Euler and Navier-Stokes equations. As computer speed increases, more and more of the physics can be simulated. This talk will discuss the current status and trends of parallel supercomputers. While hardware trends allow us to solve more complicated problems, algorithm developments are also essential. This talk will address some new methods for solving aeroacoustics problems. Most of these new developments involve hybrid schemes that couple CAA methods with integral methods or traditional CFD codes with CAA codes. Since aeroacoustics problems involve length and time scales that are beyond computer power at the present time, clever schemes must be developed for using appropriate algorithms for the various physical phenomena. One very promising scheme [J. Comput. Phys. 133 (1997)] is the nonlinear disturbance equation (NLDE) method, which uses a traditional CFD code to predict the steady-state portion of the problem, and then a higher-order method to solve for the disturbances. The steady-state portion is included in the coefficients and source terms in the NLDE. [Work supported by NASA Langley.] 
3pPA4. On the exact solution of the acoustic wave equation in a parabolic velocity profile in a hard-walled duct. L. M. B. C. Campos and J. M. G. S. Oliveira (Instituto Superior Téchnico, 1096 Lisboa Codex, Portugal)

The exact solution of the acoustic wave equation in a parabolic shear flow profile is obtained; the only exact solution given in the literature [Goldstein and Rice (1963); Jones (1977), (1978); Scott (1979); Koutsoyannis (1980)] is for a linear velocity profile, and the solution for the exponential shear flow is given elsewhere [Campos and Serrão (1998)]. The wave equation has three singularities, like the Gaussian hypergeometric equation, but it is of an extended type, since the singularity at infinity is irregular (all three singularities of the Gaussian hypergeometric equation are regular). The other two singularities of the present equation are regular, and one lies at the axis of the duct, and the other at the dicritical layers, where the Doppler-shifted frequency vanishes. The critical layers do not exist (they lie outside the duct) for longitudinal wave vector antiparallel to the mean flow (case I), i.e., propagation upstream in a local frame of reference. In the opposite case of longitudinal wave vector parallel to the mean flow (case II) there are three subcases, depending on whether the Doppler-shifted frequency on the axis of the duct is: (case II A) positive, i.e., critical layers are at imaginary "distance;"' (case II B) zero, i.e., the critical layer lies on the axis of the duct: (case II C) negative, i.e., two critical layers exist in the duct. In all cases (I, II A, II B, II C) it is possible to obtain the exact acoustic field over the whole flow region by expanding around the singularities and matching solutions. Since the acoustic wave equation in a shear flow does not lead to a Sturm-Liouville problem, the eigenfunctions need not be orthogonal or complete. There is a single set of natural frequencies and normal modes in cases I, II A, and II B, but not in case II C; in the latter case, where two critical layers lie in the flow, they may separate three sets of eigenvalues/ eigenfunctions, in the regions between by the critical layers and the walls.

5:40

3pPA5. Experimental verification of a radial mode analysis technique using wall-flush mounted sensors. Lars Enghardt (DLR-Institut fuer Antriebstechnik, Abteilung Turbulenzforschung Berlin, Mueller-Breslau-Str. 8, 10623 Berlin, Germany, lars.enghardt@dlr.de), Yanchang Zhang (LUK GmbH \& Co., 77815 Buehl, Germany), and Wolfgang Neise (DLR-Institut fuer Antriebstechnik, 10623 Berlin, Germany)

Sound fields in the inlet and outlet ducts of axial fans, compressors, and aircraft engines propagate as higher-order acoustical modes in a wide-frequency range. Decomposition of the sound field into azimuthal and radial modes permits direct conclusions on the sound generation processes and can lead to the identification of aerodynamic source areas. An established experimental method for assessing the sound field is to circumferentially traverse a radial microphone rake over 360 degrees, to measure the sound pressure in the duct at multiple circumferential and radial positions. On the inlet side of a turbo-machine this procedure cannot be employed because the wake of the rake would disturb the inlet flow conditions of the machine and alter its acoustic characteristics. Computer simulations have shown that the radial mode structure of the sound field can also be determined by measuring the sound pressure only at the inner duct wall, but at different axial locations. In this paper, experiments are described where the sound field in the outlet duct of an axial-flow fan is measured once with the conventional radial microphone rake and a second time with wall-flush mounted microphones. Subsequent radial mode analyses are carried out for both sets of measurement data to experimentally verify the new method.

\section{Contributed Paper}

6:00

3pPA6. Experimental study of acoustic impedance of a Helmholtz resonator submitted to a grazing flow. Slaheddine Frikha, Pierre Yves Hennion, and Samir Boukhari (Laboratoire de Mecanique Physique, UPMC CNRS, Upresa 7068, France, frikha@ccr.jussieu.fr)

Helmholtz resonators are usually used in ducts or in engines inlet and exhaust systems in order to reduce radiated noise. Their efficiency may be strongly affected since they are submitted to a fully turbulent grazing flow. An experimental analysis of a branched Helmholtz resonator composed by a circular neck and a circular cavity is presented here. An attempt is made to understand the evolution of entry impedance modification with respect to mean flow and the shear layer characteristics at the vicinity of the resonator aperture. An inverse method is used to identify the impedance of 25 resonators. This method presents the advantage that it is completely nonintrusive and does not require source control. The grazing flow modifies the orifice resistance and the effective neck length. A parametric study of these corrections is performed using experimental results obtained for a set of 25 resonators and a flow Mach number varying from 0 up to 0.25 . The mean flow structure is also analyzed using a numerical model. The dimensions of the tested resonators are similar to those usually used in the exhaust and inlet systems of automotive engines. That leads to a new parametric model of these corrections more suitable to engine applications. 


\title{
Contributed Poster
}

This poster will be on display in the Poster Gallery from Thursday to Friday, 18-19 March. Authors will be at their posters from 10:00 a.m. to 12:00 noon on Thursday, 18 March.

3pPA7. On the acoustic of inverse sinusoidal and catenoidal nozzles. L. M. B. C. Campos and F. J. P. Lau (Instituto Superior Técnico, 1096 Lisboa Codex, Portugal)

The acoustic wave equation, for quasi-one-dimensional propagation, along a duct of varying cross section, containing a low Mach number mean flow, is obtained using as variables either the potential or the velocity; the ray approximation, which holds only for wavelength short compared with the lengthscales or variation of cross section and mean flow velocity, is used as a factor to reduce the wave equation to a Schrödinger form. It is shown that the latter, reduced from, for the potential, is the most convenient to study the acoustics of catenoidal and sinusoidal nozzles; it is found that these inherit respectively the filtering properties of catenoidal horns, and transparency properties of sinusoidal horns. This approach also applies to the exponential nozzle whereas, for the Gaussian nozzle, the sound field can be expressed in terms of Hermite functions, using a semireduced form of the wave equations. The exact solutions of the nozzle wave equation, for the four families of ducts, are plotted as amplitude and phase versus distance, for several combinations of frequency and low Mach number. The nozzle families considered include the catenoidal in the sinusoidal and exponential and the first six eigenfunctions of Gaussian nozzles. The acoustics of inverse sinusoidal and catenoidal nozzles can also be studied, without use of the preceding transformations, via the solution of modified Mathieu equation.

\section{Session 3pPPa}

\section{Psychological and Physiological Acoustics: Perceptual Processing of Cross-Spectral Information}

\author{
John F. Culling, Chair \\ School of Psychology, Cardiff University, Cardiff CF1 3YG, UK
}

Chair's Introduction-4:15

Invited Papers

4:20

3pPPa1. Matching formant frequencies of complex sounds on different fundamentals. C. J. Darwin and Pascal Dissard (Exp. Psych., Univ. of Sussex, Brighton, Sussex BN1 9QG, UK)

In order to explore the ability of listeners to match formant frequencies in periodic sounds that differ in their fundamental frequency $(F 0)$, listeners adjusted the frequency of one formant of periodically excited one- or two-formant sounds to match the timbre of a similar target sound that could have either the same or a different $F 0$. Variability of matching (s.d. of matches within each listener) was low when matching sounds were on the same $F 0$. Variability increased when matching sounds had different $F 0$ s. However, this increase was larger for sounds with resolved harmonics than for those with unresolved harmonics. This difference may reflect the perceptual cost of estimating the spectral envelope (and formant frequency) from the excitation pattern. [Work supported by EPSRC Grant No. GR/L03422.]

$4: 40$

3pPPa2. A "missing-data" model of $\boldsymbol{F}$ 0-invariance in vowel perception. Alain de Cheveigne (LLF, Univ. of Paris 7, 2 Pl. Jussieu, Paris, France)

In steady-state voiced speech production, the vocal tract transfer function is sampled at multiples of the fundamental frequency $(F 0)$. At high $F 0$, sparse sampling causes two problems: (a) a gradual loss of information defining the spectral shape, and (b) $F 0$-dependent distortion due to aliasing. If the shape of the spectral envelope contains lag-domain components (spatial frequencies) beyond the Nyquist limit, they are folded relative to the Nyquist limit and mixed with in-band components. The Nyquist limit ( $T 0 / 2$, where $T 0=1 / F 0$ ) depends on the spacing between sampling points along the spectral envelope, and thus on $F 0$. Distortion is thus $F 0$-dependent, and all the more severe as $F 0$ is high. Smoothing or interpolation are ineffective in dealing with this problem, and cannot produce an $F 0$-invariant pattern. A solution is proposed based on the concept of "missing feature theory," recently proposed for speech recognition. Pattern matching is limited to available data ( $F 0$-spaced sample points) using an $F 0$-dependent weighting function. All other points are ignored. The model is proposed in two versions, one that operates on the short-term spectrum or excitation pattern, the other on the autocorrelation function. The model ensures $F 0$-independent vowel identification, at the cost of an $F 0$ estimate. 
3pPPa3. Evidence of a dissociation between the mechanisms governing the perceptual grouping of spectral components and the computation of low pitch. Brian Roberts and Jeffrey M. Brunstrom (School of Psych., Univ. of Birmingham, Edgbaston, Birmingham B15 2TT, UK, b.roberts@bham.ac.uk)

The perceptual fusion of harmonically related partials is often assumed to result from the operation of a template mechanism that is responsible for computing low pitch. This dual-role hypothesis has been tested using frequency-shifted complexes. These sounds are inharmonic, but preserve a regular spectral pattern of equal component spacing. Stimuli had a nominal fundamental $(F 0)$ frequency of $200 \mathrm{~Hz}$ (roving range $\pm 20 \%$ ), and were frequency shifted either by $25.0 \%$ or $37.5 \%$. Three consecutive components (6-8) were removed and replaced with a sinusoidal probe, located at one of a set of regularly spaced positions spanning the gap. On any trial, subjects heard a complex tone followed by an adjustable pure tone in a continuous loop. Subjects were well able to match the pitch of the probe unless it corresponded with a position predicted by the spectral pattern of the complex. In contrast, hit rates were not depressed for probes positioned at integer multiples of the $F 0$ (s) corresponding to the low pitch(es) of the complex, which were predicted from previous data [Patterson, J. Acoust. Soc. Am. 53, 1565-1572 (1973)]. The findings suggest that separate mechanisms are responsible for the computation of low pitch and for the perceptual grouping of partials.

\section{$5: 20$}

3pPPa4. Auditory processing of coherent and incoherent frequency modulation. Robert P. Carlyon (MRC Cognition and Brain Sci. Unit, 15 Chaucer Rd., Cambridge CB2 2EF, England, bob.carlyon@mrc-cbu.cam.ac.uk)

When two elements of a visual array move coherently, there is a strong tendency to group them perceptually. This has prompted several authors to suggest that acoustic frequency components which undergo coherent FM should be perceptually fused, and that those which are modulated incoherently should be segregated. This paper will review the mounting body of evidence demonstrating that, once a number of co-varying cues are controlled for, the auditory system is insensitive to the difference between coherent and incoherent FM. Such cues include the greater inharmonicity, smaller swings in average frequency, and more salient distortion products which can occur with incoherent compared to coherent modulation. In addition, new evidence will be presented showing that listeners can be more sensitive to one form of incoherent FM than to another: Opposite-direction quasi-linear frequency sweeps imposed on two carriers are more detectable when the frequency of the lower component decreases and that of the higher component increases than vice versa. This occurs even though the frequency sweeps imposed on the individual components are equally detectable when those components are presented in isolation, and even though within-channel interactions are masked by noise.

\section{Contributed Papers}

$5: 40$

3pPPa5. Searching for a time window for timbre: Dynamic spectral profiles. M. E. H. Schouten and W. J. M. Peeters (Res. Inst. for Lang. and Speech UiL-OTS, Utrecht Univ., Trans 10, 3512 JK Utrecht, The Netherlands, bert.schouten@let.uu.nl)

Subjects were asked to match resonance glides, of various durations and sweep widths around $1000 \mathrm{~Hz}$, with two connected steady-state resonances whose frequencies could be controlled independently. The expectation was that short resonance glides (below $100 \mathrm{~ms}$ ), would give rise to single, apparently stationary, perceptual events, so that the two matching steady-state patterns would not rise or fall. Long glides, on the other hand, were expected to be perceived as rising or falling and to be matched accordingly. It was hoped that this would provide a basis for an explanation of diphthongs as unitary percepts. The resonances formed part of dynamic spectral profiles and consisted of single formantlike peaks swept along the top of a spectral base, consisting of 25 harmonically or logarithmically spaced components from 200 to $5000 \mathrm{~Hz}$. There was an effect of duration, although it interacted with sweep width. At durations shorter than about $120 \mathrm{~ms}$, subjects placed the two steady profiles that they had to match with the dynamic profile, much closer together than with durations over $120 \mathrm{~ms}$; however, this only occurred if sweep width was more than $600 \mathrm{~Hz}$.

\section{6:00}

3pPPa6. A spectral layer model in speech and music perception. Werner A. Deutsch (Austrian Acad. of Sci., Liebiggasse 5, A-1010, Vienna, Austria,wad@kfs.oeaw.ac.at)

According to a model of the peripheral hearing system establishing a masking and overmasking paradigm, speech and music signals have been split into several spectral layers by means of spectral subtraction. Spectral components below the so-called psychoacoustical irrelevance threshold are eliminated in a first processing step. The resulting signal, which exhibits no noticeable difference to the unprocessed one, is fed into an overmasking procedure applying flattened hearing masking functions. This action implements a kind of a spectral peak detector device. Consequently, the discrimination between spectral peaks (auditory figure) and background components, especially in reverberant environments, is improved. As a result, different audible signal parts according to different spectral layers are obtained, separating spectral peaks and weaker spectral components. In a certain range, both parts of speech and music signals are equally intelligible. In music, leading voices can be extracted and separated from the orchestra sound. Recent research indicates that this type of figure-background discrimination based on the dynamic characteristics of the acoustic signal only can exhibit several perceptually relevant features in listeners with normal hearing as well as in subjects with sensineural hearing impairment. 


\title{
Session 3pPPb
}

\section{Psychological and Physiological Acoustics: Sound Quality}

\author{
Gerhard Krump, Chair \\ Harman Audio Electronic Systems GmbH, Schlesische Strasse 135, D-94315 Straubing, Germany
}

\section{Contributed Papers}

$4: 20$

3pPPb1. Interior car sound quality-Assessment of acceleration noises. Reinhard Weber (Dept. of Phys., Carl-von-Ossietzky Univ., D-26111 Oldenburg, Germany)

Investigation of booming noise effects was the reason for the present study. Interior noises of six different cars accelerating from 1500 to 6000 rpm at third gear (full load) were presented over headphones. Seventy normal-hearing subjects (approximately one-third males and two-thirds females) aged from 20 to 60 years (at least 15 subjects per decade) took part in two experiments. After an initial orientation phase, preference judgments were made using a paired comparison paradigm. In experiment 2, the noises were assessed on a bipolar categorical scale (disagreeable) agreeable) with nine subdivisions. Immediately after the experiments, an interview was conducted to learn about the subjects' impressions about the noises. The preference data were analyzed using MDPREF and compared to the categorical judgment data for different subsets of subjects. The results on subjective data will be presented, together with a correlation analysis on their relationship with a number of acoustical and psychoacoustical signal parameters (level, loudness, sharpness, fluctuation strength, roughness).

\section{4:40}

3pPPb2. The influence of sound quality on expected visual impression. Monica M. Söderholm (Electrolux Resesearch \& Innovation, $\quad \mathrm{S}-105 \quad 45 \quad$ Stockholm, monica.soderholm@notes.electrolux.se) and Daniel Västfjäll (Chalmers Univ. of Technology, S-412 96 Göteborg, Sweden)

Information from visual and auditory systems is often linked. One example of the cross-modal connection between sound and visual experience is different materials that easily can be identified by their sound quality. The purpose of this investigation was to analyze the anticipated visual impression of three vacuum cleaners when listening to the product. The participants in the listening test where asked to describe the visual impression of the vacuum cleaners and also how they thought the vacuum cleaner sounded like with own words. The 24 listeners were both female and male of different ages and the vacuum cleaners were judged live. Often something that is large, for instance, a truck compared to a car, does produce more sound. The experience that large products are louder than small products is an influence when listening to vacuum cleaners. The listeners expected a large vacuum cleaner when listening to a rather loud vacuum cleaner. There was a unanimous opinion among the participants that a loud vacuum cleaner was ugly, had a box shape, and was old. The vacuum cleaners that were described to have a nice sound were described as small and round with a nice design and nice colors.
5:00

3pPPb3. Comparison of two semantic-differential test methods. Etienne Parizet (Renault, 67 rue des bons raisins, 92508 Rueil-Malmaison Cedex 9, France, etienne.parizet@renault.com)

Two methods for conducting a multidimensional test were investigated. In the first and classical one, a sound (or a pair of sounds) is presented to the subject, who has to evaluate it (or compare the two sounds) for the various semantic parameters. In the second one, the test is repeated for each parameter (i.e., subjects must evaluate the whole set of sounds for the first parameter, then again for the second one, and so on). These two methods were compared on the basis of five diesel engine noises in small cars driving at idle. Sounds were compared in pairs for seven parameters (pleasant, loud, etc.). The characterization of sounds thus obtained was similar for the two methods. The second one proved to be more accurate (the accuracy is evaluated on the basis of repetition and triadic errors) and with a shorter duration than the first one, because listeners are concentrated on the selected sound parameter while hearing a new stimulus. Therefore, this method is recommended for conducting semantic differential tests.

\section{$5: 20$}

3pPPb4. About perceptivity of narrow-band frequency response variations of loudspeakers. Gerhard Krump (Harman Audio Electron. Systems GmbH, Schlesische Str. 135, D-94315 Straubing, Germany, gkrump@harman.de)

Many loudspeakers show peaks and gaps at $1 \mathrm{kHz}$ caused by the connection of the hard cone and soft surround, which produces interferences and complex vibrational modes. Great efforts are made by the speaker manufacturer to avoid this by changing the surround geometry and material, whereas primarily knowledge about the perceptive limits of the peaks and gaps in frequency response would be necessary. For this investigation line spectra with equal amplitudes and statistical phases between 1 $\mathrm{Hz}$ and $20 \mathrm{kHz}$ were calculated by a personal computer. At $1 \mathrm{kHz}$ spectral enhancements or gaps with a bandwidth of $1 \mathrm{~Hz}$ or of a half, one, or two critical bands were realized with five different critical band levels. Ten test persons had to compare the first line spectrum with equal amplitudes (anchor) with the second signal with spectral changes in a yes-no procedure presented by an earphone with free-field equalizer at a SPL of $70 \mathrm{~dB}$. The just-noticeable changes in spectral envelope are different for enhancements and gaps and depend on the bandwidth and the critical band level of the variations. They can be explained with calculated masking patterns and literature data. 
3pPPb5. Determination of hearing properties and their connection to psychoacoustical parameters. Kai Johannsen and Holger Prante (Inst. of Tech. Acoust., Einsteinufer 25, 10587 Berlin, Germany, johannsen@mach.ut.tu-berlin.de)

The sound quality of products is often evaluated in hearing experiments using the semantic differential and a subsequent factorial analysis. In this study the method is applied to determine basic hearing properties. In order to achieve optimal results of the hearing experiment, special interest has been taken in the choice of adjectives and sounds. This selection is based on the results of a previously conducted survey of sound attributes. The selected 25 sounds were recorded using an artificial head and presented binaurally to a jury of 20 adults with normal hearing. A factorial analysis of the ratings on 24 attribute scales produced six factors representing basic perceptive cognitive and emotional hearing dimensions. In a last step connections between these dimensions and psychoacoustical parameters calculated from the sounds were evaluated using an analysis of correlation. Although the results show high correlations between loudness, sharpness, and pitch strength and equivalent attributes, no such connections could be found for the psychoacoustical roughness and fluctuation strength. In order to find better-correlated parameters, other calculation methods for these properties are investigated.
3pPPb6. New approach in measuring auditory source width. Jörg Becker, Markus Sapp, and Frederik Görges (Inst. of Commun. Eng., Aachen Univ. of Technologie, 52056 Aachen, Germany)

Depending on the acoustical surrounding sound sources appear to be broadened. This spatial impression can be rated by a quantity called auditory source width. In this paper a new method for determining this auditory source width is presented. The system is based on the reproduction of an artificial head recording of the examined sound field mixed with a pair of virtual acoustic pointers. These pointers can be moved by test persons to fit the left and right border of the sound source whose ASW has to be rated. The acoustical pointers are implemented as digital signal generators followed by real-time HRTF filters, measured in azimuth steps of $2 \mathrm{deg}$ and cross faded between the directions. Investigations on suitable temporal and spectral shapes of the pointer signals have been made to minimize the localization blur of the pointer. The HRTF measurements and the recordings were done with different artificial heads in order to examine their influence on the determined ASW. Furthermore the influence of different sound field reproduction systems-headphone and crosstalk cancellation-are examined.

WEDNESDAY AFTERNOON, 17 MARCH 1999

ROOM H2036, 4:20 TO 6:20 P.M.

\title{
Session 3pSA
}

\section{Structural Acoustics and Vibration: Structural Vibrations}

\author{
Jacek Jarzynski, Cochair \\ George W. Woodruff School of Mechanical Engineering, Georgia Institute of Technology, Atlanta, Georgia 30332-0405, USA \\ David Feit, Cochair \\ Carderock Division, Naval Surface Warfare Center, 9500 MacArthur Boulevard, West Bethesda, Maryland 20817, USA
}

\section{Contributed Papers}

4:20

3pSA1. Torsional waves in a viscoelastic layer. Qingchun $\mathrm{Li}$ and Jacek Jarzynski (The George W. Woodruff School of Mech. Eng., Georgia Inst. of Technol., Atlanta, GA 30332-0405, jacek.jarzynski@me.gatech.edu)

The objectives of this study are first to examine experimentally the propagation of torsional waves in a viscoelastic material, and second to use these data to determine the complex shear modulus of the material. The viscoelastic material used in this study is neoprene rubber. A magnetic drive is used to generate a transient, one-cycle, torsional wave in a brass rod, and this wave is transmitted into a layer of neoprene rubber bonded to the rod. A fiber-optic laser Doppler vibrometer is used as a noncontact probe to measure the torsional wave transmitted through the neoprene layer. The experimental data are compared with predictions from a theoretical model [D. P. Thomas, Int. J. Eng. Sci. 6, 565-570 (1968)]. Also, the complex shear modulus is determined from a least-square fit of the theoretical model to the experimental data using the downhill Simplex method. Another application of torsional waves is to nondestructive testing of materials, particularly for testing the strength of adhesive bonds between layers. [Work supported by ONR.]
4:40

3pSA2. Estimates of dynamic strain and stress in pipes by measured average vibration velocity. Svante Finnveden (Dept. of Vehicle Eng., KTH, SE-100 44 Stockholm, Sweden, svantef@fkt.kth.se) and Roger Pinnington (Univ. of Southampton, Southampton SO17 1BJ, England)

A velocity method for estimating dynamic strain and stress in pipe structures is investigated. With this method predicted or measured spatial average vibration velocity and theoretically derived strain factors are used to estimate maximum strain. The nondimensional strain factor is defined as the maximum strain times the ratio of the sound velocity to the spatial rms vibration velocity. Measurements are made confirming that this is a descriptive number. Using a spectral finite-element method, numerical experiments are made varying the pipe parameters and considering all 16 possible homogeneous boundary conditions. While indicating possible limitations of the method when equipment is mounted on pipes, the experiments verify the theoretical results. The velocity method may become useful in engineering practice for assessments of fatigue life. 
3pSA3. On the dynamic interaction of a pipe conveying fluid with an elastic resisting medium. Ventzeslav A. Dzhupanov, Peter A. Djondjorov, and Vassil M. Vassilev (Inst. of Mech., Bulgarian Acad. of Sci., Sofia, Bulgaria)

The problem of the dynamic stability of pipes conveying high-speed fluid and lying on an elastic foundation has been a subject of significant interest during the past 30 years due to the broad application of pipes as structural components in different technologies. The present study contributes to the solution of this problem, suggesting a new model of the foundation - an infinitely deep elastic stratum, whose width is equal to the length of the attached pipe. The material of the foundation is supposed to be a homogeneous and isotropic one. Its dynamic behavior is considered in the context of linear elasticity. The pipe vibration is governed by the usual Bernoulli-Navier-Mohr theory. An approximate solution of the respective mathematical problem is obtained applying Galerkin's method. As a result, the influence of the medium physical characteristics to the flutter dynamic stability of cantilevered pipes through the pair of acoustic velocities is examined.

\section{$5: 20$}

3pSA4. Investigation at a top counter weight of a washing machine. Ulrich Heise (Noiselab, FHP Motors GmbH, Klingenbergplatz 1, 26133 Oldenburg, Germany) and Wolfgang Harbich (Noiselab, 13599 Berlin, Germany)

During the development of a new washing machine a problem came up in spite of the durability of the newly designed top counter weight. The top counter weight is necessary to balance the drum of the machine. The problem was caused by the dynamical behavior of the system. A modal analysis was made at the compensation weight in the machine. Several different modes and eigenvalues were detected. The disturbing mode was found. It was caused by the stiffness of the fastening and the mass of the weight. The top counter weight was fastened in the axial direction of the drum. The disturbing mode was eliminated due to the change of fastening from axial to radial fastening. The problem is now solved. Another point of view are the other eigenvalues of the weight. The size of the weight looks like a xylophone bar and, in the same manner, it can vibrate. These modes of the weight itself are not disturbing modes, because the excitation forces are too small in this area and at this frequency range for exciting the compensation weight.
3pSA5. A mixed use of finite element and geometrical techniques. Philippe Jean (Centre Scientifique et Technique du Bâtiment, 24 rue Joseph Fourier, 38400 St. Martin d'Hères, France)

The finite-element method is limited to either small geometries or to the low-frequency range, whereas geometrical approaches are best applied at medium and high frequencies, but do not take vibrating surfaces into account. When the vibrating boundaries of a volume are only a small part of the total boundary, it is worth combining the two methods. By using geometrical methods to compute a Green's function that includes all wave reflections on the surfaces of the volume, it is possible to restrict the integral representation of the pressure field to the vibrating surfaces. The surfaces to be meshed will be reduced to surfaces with known velocity fields. This approach offers a means of reducing computation times. It has been applied to large volumes corresponding to a room with a vibrating concrete wall and to a train with a vibrating window.

\section{6:00}

3pSA6. Irregular modes at the endshield of a heating system motor. Ulrich Heise (Noiselab, FHP Motors GmbH, Klingenbergplatz 1, 26133 Oldenburg, Germany)

Nowadays, more and more modern heating systems are not installed in separated rooms, so the expectation of noise comfort has been increased for the last years. A noise and vibration optimization was made at an electric motors b-sided endshield. Sometimes, the old endshield emitted airborne noise. This was caused by the structure-borne noise of the bearings together with a mode of the endshield. A modal analysis was made to detect the disturbing mode. The amazing thing of this test was that the $(0,1)$ mode frequency has a higher resonance frequency as the $(1,1)$ mode. Normally the $(1,1)$ mode frequency has a 1.594 higher frequency than the $(0,1)$ mode resonance [N. H. Fletcher and T. D. Rossing, The Physics of Musical Instruments (1991), pp. 69-71]. The b-sided bearings can make a mass coupling from the rotor to the endshield in radial direction, but not in this way in the axial direction. The $(1,1)$ mode of the endshield had nearly the same structure-borne amplitude as the $(0,1)$ mode, but the $(1,1)$ mode does not transmit airborne noise. This depends on the mechanical dimensions of the $(1,1)$ mode, because this mode is working with its dimensions on an acoustical short circuit on air. 


\title{
Session 3pSC
}

\section{Speech Communication: Psychoacoustic Methods in Speech Recognition II}

\author{
Birger Kollmeier, Cochair \\ AG Medizinische Physik, Universität Oldenburg, Postfach 2503, D-26111 Oldenburg, Germany \\ Hynek Hermansky, Cochair \\ Oregon Graduate Institute of Science and Technology, Beaverton, Oregon 97006-8921, USA
}

Chair's Introduction-4:15

Contributed Papers

4:20

3pSC1. Application of a roughness model to pathological voices. Robert Reuter (Inst. Electron., Techn. Univ., Einsteinufer 17, D-10587 Berlin, Germany, rreuter@tubife1.ee.tu-berlin.de) and Hanspeter Herzel (Humboldt Univ., D-10115 Berlin, Germany)

Irregularities in voiced speech are often related to the intrinsic nonlinearities in the vibrations of the vocal folds. Desynchronization of vibratory modes can induce nonlinear phenomena such as subharmonics, biphonation (two independent pitches), and deterministic chaos. The resulting complex signals exhibit various amplitude and frequency modulations and are, therefore, perceived typically as rough voices. In clinical studies of pathological voices perceptive roughness scores are widely used. So far, however, no satisfactory correlation of ratings of roughness and acoustic parameters has been found. In this presentation a modified Aures model for the quantification of roughness is introduced. It is exemplified that subharmonics lead to high roughness scores whereas the roughness of biphonation depends strongly on the two frequencies. Finally, a set of 120 perceptually evaluated pathological voices is studied. It turns out that the roughness score obtained from the psychoacoustic model is stronger correlated to the ratings of phoniatricians than conventional perturbation measures such as jitter and shimmer.

\section{$4: 40$}

3pSC2. How children recognize spoken words in speech recognition threshold assessment. Lebel Caroline (Institut Raymond-Dewar, 3600 Berri, Montreal, QC H2L 4G9, Canada) and Picard Michel (Universite de Montreal, Montreal, QC H3C 3J7, Canada)

The purpose of this study was to investigate the potential influence of language context effects on the speech recognition threshold (SRT) in children of different ages. To determine the respective contributions of audibility and linguistic factors to the SRT, the speech intelligibility index (SII), and indices $k$ and $j$ as proposed by Nittrouer and Boothroyd [J. Acoust. Soc. Am. 87, 2705-2715 (1990)] were calculated for the following conditions: (1) SRT using an open-set response format; (2) SRT using a closed-set response format (dissimilar foils to increase predictability); and (3) SRT using test words at the beginning of a short sentence. Listeners were three groups of 24 normally hearing children in the first, third, and fifth grades, respectively. Results indicated that SII and factor $j$ decreased significantly and factor $k$ increased proportionally as a function of both lexical and sentence-level context effects in all groups. This suggests that audibility factors are less critical in the facilitating presence of language contexts. The largest effect was shown for lexical context effects with predictable outcome of decreased SRTs. These findings are compelling evidence of language context effects playing a significant role in school-age children required to recognize speech at barely audible levels.
5:00

3pSC3. Silicon cochlea: A digital VLSI implementation of a quantitative model of the auditory system. Matthias Brucke, Wofgang Nebel (Univ. of Oldenburg, Computer Sci. Dept., VLSI Group, 2611 Oldenburg, Germany), Alexander Schwarz, Bärbel Mertsching (Univ. of Hamburg, Hamburg, Germany), Martin Hansen, and Birger Kollmeier (Univ. of Oldenburg, Oldenburg, Germany)

In this paper a digital VLSI implementation of a quantitative model of the auditory system is presented, which includes several processing stages physiologically and psychoacoustically motivated by the function of the human ear. The model was successfully applied to a wide range of applications from psychoacoustical experiments to speech recognition. The hardware design is derived from a $\mathrm{C} / \mathrm{C}++$ notation of the algorithms using floating point arithmetics. One application of the model is used to determine the necessary internal precision of the computations for a transfer into a version suitable for a hardware implementation using fixpoint arithmetics. This application provides a very valid test bench since the significant speech processing features of the model are proofed by computing an objective speech quality measure. The processing scheme is divided into designs for two FPGAs and will be converted into ASICs in a later version. The implementation of the model as dedicated hardware provides efficient coprocessing power and allows implementations of complex auditory-based speech processing algorithms in real time because the chipset performs substantial parts of the necessary calculations.

$5: 20$

3pSC4. Entropy rate based speech segmentation. Wolfgang Wokurek (Inst. of Natural Lang. Processing, Exp. Phonet., Stuttgart Univ., Azenbergstrasse 12, Stuttgart, 70174, Germany, wokurek@ims.uni-stuttgart.de)

The sequence of estimates of the speech signal's entropy rate is investigated as a potential basis for speech segmentation. Raising and falling edges of that entropy rate curve and its maxima and minima are considered as candidates for segment boundaries. These prominent points are compared to the phonetic segment boundaries and to acoustic landmarks [S. A. Liu, J. Acoust. Soc. Am. 100, 3417-3430 (1996)]. The comparison is made using the "Kiel corpus of read speech" which is a phonetically manually labeled corpus of German utterances. The evaluation considers the entropy rate labels against the background of the phone segments. In particular the position and the count of the entropy rate labels within each phone are appraised. The average results are reported both as totals and broken down into phone classes and phones. The frame duration of the entropy rate estimation controls the smoothness of the curve and hence the label count per phone. A similar evaluation relates the entropy rate labels to the acoustic landmarks. 
Session 3pSP

\title{
Signal Processing in Acoustics and Engineering Acoustics: Binaural Technology II
}

\author{
Mahlon Burkhard, Cochair \\ Sonic Perceptions, Inc., 6964 Kennsington Road, Brighton, Michigan 48116, USA \\ Jens Blauert, Cochair \\ Communication Acoustics, Ruhr-Universität Bochum, D-44780 Bochum, Germany \\ Chair's Introduction-4:35
}

Contributed Papers

\begin{abstract}
4:40
3pSP1. Implementation issues in the development of a real-time, Windows-based system to study spatial hearing. Joel D. Miller (Raytheon, NASA Ames Res. Ctr., M.S. 262-6, Moffett Field, CA 94035-1000, jdmiller@mail.arc.nasa.gov), Jonathan S. Abel (San Jose State Univ., NASA Ames Res. Ctr., Moffett Field, CA 94035-1000), and Elizabeth M. Wenzel (NASA Ames Res. Ctr., Moffett Field, CA 94035-1000)
\end{abstract}

As a tool to study spatial hearing, a customizable real-time 3-D-audio and virtual acoustic environment rendering system, Sound Laboratory (SLab), was developed. Design goals for this system included flexibility, extensibility, maintainability, and a fast development cycle per experiment. SLab is a Windows application composed of five distinct software layers: Application programming interface (API), 3D projection, signal flow translation, signal routing and processing, and digital signal processing library. The acoustic scene, including listener, source, and environment characteristics, is specified in the API layer and translated in the 3-D projection layer to geometric quantities, such as range and arrival angle, for each path rendered between the source and listener. The geometry and head-related transfer function coefficients are rendered using a set of parallel finite impulse response filters and delay lines built on Intel's Signal Processing Library. This rendering architecture provides the flexibility and the extensibility required for psychoacoustic experimentation, while the modular nature of the programming interface ensures maintainability and quick development of new experiments. The psychoacoustic parameters to be manipulated, the signal flow diagram, and the five software layers are discussed in detail, and various implementation issues are examined.

\section{5:00}

3pSP2. Computational model of an individual head-related transfer function using the BEM. Brian F. G. Katz ${ }^{\mathrm{a}}$ (Acoust. Dept., Penn State Univ., State College, PA 16801)

The head-related transfer function (HRTF), determined by the complex geometry of the pinnae and head, is the primary research and commercial tool for exploring and exploiting 3-D auralization and auditory localization. Research into the determining characteristics of the HRTF is difficult, as the anatomical variations between individuals are many. These variations make comparisons between individuals less than ideal for determining the relation between structure of the individual and components of the HRTF. This paper will discuss an investigation into the use of a geometrical model of an individual to calculate the HRTF. The calculation utilizes the boundary element method (BEM). As a tool, the use of a computer model allows for selected variations in the individual's anatomy and direct observation of the results, a difficult task in experimental mea- sures. Previous work by the author discussed the variability in experimental measurement techniques of the HRTF. Comparisons are made between the model results and these HRTF measurements for the same individual. Some preliminary variations to the model and their effects are also discussed. Computational results show good agreement with the general trends of the measurements and the frequency limitations of the computation. ${ }^{\text {a) }}$ Currently at Artec Consultants, Inc., 114 W. 26th St., New York, NY 10001

$5: 20$

3pSP3. Auditory perception of reverberant surroundings. Jesper Sandvad (Waisman Ctr., Univ. of Wisconsin, Madison, WI and Acoust. Lab., Aalborg Univ., Denmark)

Three experiments on perception of reverberant surroundings were performed. In the first experiment, listeners were presented with binaural recordings of a speech signal and a set of photographs taken in different rooms, and were asked to point out where each recording was made. Most listeners performed very well in this task (average number of correct answers was 70\%), showing that reverberation contains information on room properties that the listeners are able to extract. In the second experiment, listeners were asked to judge the relative size of different rooms by listening to the recorded signals. The results show substantial individual differences, with some listeners being close to veridical performance while others had only a few correct answers. A scaling analysis suggests that some listeners use the direct/reverberant energy ratio, while others use reverberation time as a cue for room size. In the third experiment, artificial reverberation generated by a geometric room model (including individual reflections) and from a stochastic model (controlling only energy measures) were compared with signals generated directly from measured room impulse responses. The results show comparable performance, suggesting that energy measures are the most important for assessing information on room parameters. [Work supported by ONR Grant No. N00014-96-10675.]

\section{$5: 40$}

3pSP4. Comparison of wideband LMS, subband LMS, and a nonlinear entropic neural approach to adaptive noise cancellation for speech enhancement. P. W. Shields, M. Girolami, and D. R. Campbell (Dept. of Electron. Eng. and Phys., Univ. of Paisley, High St. Paisley, Renfrewshire PA1 2BE, Scotland, paul@diana22.pailsey.ac.uk)

An empirical comparison of three adaptive algorithms for speech enhancement in noisy reverberant conditions is presented. The subband least mean square (LMS) [E. Toner and D. R. Campbell, Speech Commun. 12, 
253-259 (1993)] and minimum entropy noise reduction schemes [M. Girolami, Electron. Lett. 33(17), 1437-1438 (1997)] are compared with a reference wideband LMS approach [S. D. Stearns and R. A. David, Signal Processing Algorithms (Prentice-Hall, Englewood Cliffs, NJ, 1988)]. All three methods were applied to the enhancement of speech corrupted with speech-shaped noise. The schemes were tested using simulated anechoic and real-room reverberant $\left(T_{60}=0.3 \mathrm{~s}\right)$ environments. The anechoic and real-room recordings were binaural including head shadow and diffraction effects. The subband LMS adaptive processing scheme uses the LMS adaptive noise cancellation algorithm in frequency-limited subbands. The inputs from each microphone are split into 16 contiguous subbands using a cochlear distribution according the function provided by Greenwood [J. Acoust. Soc. Am. 87, 2592-2605 (1990)]. Each frequency-limited subband is processed using a LMS adaptive noise cancellation filter operating in an intermittent or continuous mode depending on input signal characteristics. The neural network approach is motivated by temporally sensitive hebbian super-synapses, which may form sparse representations based on commonly occurring spatio-temporal input patterns. The results for real-room signals demonstrate statistically equivalent performance of the subband LMS and wideband neural method, both of which prove superior to the wideband LMS algorithm.
6:00

3pSP5. Evaluation of the air particle velocity signal from the binaural pressure impulse response. Domenico Stanzial (CIARM-CNR c/o Cemoter Acoust. Dept., Via Canal Bianco 28, I-44044 Cassana, Ferrara, Italy) and Davide Bonsi (CIARM c/o Univ. of Ferrara, I-44100 Ferrara, Italy)

From the Lagrangian theory in linear acoustics one clearly infers that the generalized momenta of the sound field are the acoustic pressure and the three components of the air particle velocity. This means that only through the knowledge of the full set of these four quantities a complete physical identification of the sound field can be achieved. The binaural technology represents an important tool for this goal, since it offers a method for reconstructing the pressure sound field by means of a couple of pressure impulse responses measured in two close points. An additional improvement of the binaural signal processing can be obtained using the two pressure input signals for evaluating the velocity component along the direction determined by the measurement points. First the velocity impulse response component is evaluated by means of the Euler equation and then, by convolving this signal with the usual anechoic signal, the velocity is reconstructed. In this paper the rigorous mathematical procedure for accomplishing the above task is presented.

WEDNESDAY AFTERNOON, 17 MARCH 1999

ROOM H104, 4:40 TO 6:20 P.M.

\title{
Session 3pUWa
}

\section{Underwater Acoustics: Sediment Acoustics}

\author{
F. Michael Pestorius, Cochair \\ Applied Research Laboratories, University of Texas, Austin, Texas 78713-8029, USA \\ Nicholas G. Pace, Cochair \\ SACLANT Undersea Research Centre, Viale S. Bartolomeo 400, 19038 La Spezia, Italy
}

Contributed Papers

\section{4:40}

3pUWa1. Volume scattering realizations from ocean sediment soundspeed and density inhomogeneities. Kevin D. LePage (SACLANT Undersea Res. Ctr., Vl. S. Bartolomeo 400, 19138 La Spezia, Italy) and Henrik Schmidt (MIT, Cambridge, MA 02139)

Synthetic scattered field calculations can serve as a useful tool for evaluating the practicality of estimating bottom scattering properties from in-situ measurements. Here the temporal and angular characteristics of early time reverberation from volume inhomogeneity scatterers in the subbottom are investigated using perturbation theory in the framework of a spectral integral approach. In order to model realistic sediments more accurately, the theory treats the scattering from both sound-speed and density fluctuations in the presence of a background sound-speed gradient. To allow the simulation of realistic experimental geometries, the scattered field is propagated to various receivers through a general multilayered waveguide. Results show that there are several distinctive characteristics of the fields scattered by the different mechanisms which depend both on the scattering properties of the bottom and the unperturbed properties of the overall waveguide.
5:00

3pUWa2. In-sediment coherence measurements in a very shallowwater area. Joseph L. Lopes, Iris C. Paustian, and John S. Stroud (Coastal Systems Station, Naval Surface Warfare Ctr., Code R21, 6703 W. Hwy. 98, Panama City, FL 32407-7001)

A measurement was conducted in a very shallow-water (VSW) region to investigate acoustic propagation into a sandy bottom. The measurement utilized several acoustic sources and two identical hydrophone arrays. The sources were mounted onto a stationary sonar tower complete with horizontal pan and vertical tilt motors. Each hydrophone array consisted of four transducers that were buried via water-jetting methods approximately $10 \mathrm{~m}$ from the sonar tower. One array was situated such that the projected acoustic beam was parallel to the rippled structure associated with the sandy bottom, while the other was located where the transmitted beam was perpendicular to the rippled structure. Results of the in-sediment coherence data will be presented as functions of hydrophone burial depth and range in the frequency range between 30 and $150 \mathrm{kHz}$ for the two buried arrays. 
The main physical reasons preventing the generalization of bubbly liquid acoustics in this case are that bubble expansion in unlimited space differs from bubble expansion in porous media, and the most delicate moment of this generalization is how to define the mixture pressure, what the mixture pressure is, and how it corresponds to pressure in the bubbles and relates to bubble dynamics. The goal of this communication is to show how to generalize the bubbly liquid acoustics in the water-bubbles-sand case with the help of the cell-model approach. The cell model is used for the Rayleigh equation derivation for a single bubble dynamics in a porous medium, and the governing equations for nonlinear acoustic waves are derived. The obtained canonical equations can explain some experimental data of three-phase media acoustics.

\section{6:00}

3pUWa5. A mixed wave finite-element approach for solving Biot's poroelasticity equations in acoustics. Franck C. Sgard (LASH, DGCB URA CNRS 1652, ENTPE-Rue Maurice Audin, 69518 Vaulx-en-Velin Cedex, France, Franck.Sgard@entpe.fr), Noureddine Atalla, and Raymond Panneton (Univ. de Sherbrooke, QC J1K 2R1, Canada)

The use of finite-element models for solving Biot's poroelasticity equations often leads to large frequency-dependent systems. Recently, the authors have investigated the use of a selective modal analysis to decrease the size of large finite-element models involving poroelastic structures. The method used a dual real modal basis associated with the skeleton in vасио and the fluid phase occupying the volume of the porous material to approximate the $\{U, p\}$ variables. However, this method converges poorly due to the importance of the damping brought in by the porous material. In this paper, an alternate reduction technique, based on a plane waves expansion method combined with a finite-element approach, is investigated in the case of a single porous layer. The theory behind the technique, together with numerical examples illustrating the performance of the approach, will be presented. [Work supported by Centre Jacques Cartier.]

WEDNESDAY AFTERNOON, 17 MARCH 1999

ROOM H2053, 4:20 TO 6:20 P.M.

Session 3pUWb

\title{
Underwater Acoustics: Scattering from Underwater Structures
}

\author{
George V. Frisk, Chair \\ Woods Hole Oceanographic Institute, Woods Hole, Massachusetts 02543, USA
}

\section{Contributed Papers}

$4: 20$

3pUWb1. Influence of sound-structure interaction on backscattering amplitude in underwater acoustics. H. Peine (FWG, FB 331, Klausdorfer Weg 2-24, 24148 Kiel, Germany)

Sea-based backscattering experiments were carried out in the frequency range from Helmholtz numbers $(k a) 10$ to 30 with a model target. In order to improve target identification, focus here is on separating features related to sound structure interaction from those determined by the target's shape. At the frequencies under concern generally many overlapping vibration modes of the target are excited. Whereas with specially designed objects, like thin-walled hollow cylindrical or spherical shells, isolated resonance modes can be directly observed in the echo and assigned to surface waves, the model target used here resembles the complex structure of real obstacles. The structural responses measured by accelerometers attached inside the model are recorded simultaneously together with the backscattering amplitude allowing immediate comparison. Narrow-band swept sine and $\mathrm{CW}$ pulses were used. At each frequency the target was turned around a fixed axis with angle resolution better than 2 degrees. A novel spectral transfer function is tested modeling the frequency-dependent boundary impedance and therefore the envelope deformation of the scattered pulse due to interaction of the impinging wave with the target's interior. This model function has rather few parameters to be adjusted.

4:40

3pUWb2. A perturbation theory for scattering from targets near rough surface sediments: Numerical results. Garner C. Bishop and Judy Smith (Naval Undersea Warfare Ctr., Div. Newport, Newport, RI 02840)

A null field $T$-matrix perturbation theory developed for plane-wave scattering from a fluid loaded elastic spherical shell near a rough fluid elastic interface [J. Acoust. Soc. Am. 101, 767-788 (1997)] is generalized to include scattering from spherical, spheroidal, and endcapped cylindrical targets near rough surface rigid, soft, fluid, and elastic sediments. Numerical results are obtained that demonstrate some of the effects of surface roughness on resonant and nonresonant target-boundary scattering. 
3pUWb3. Localized modes of oscillating structures which lie on the bottom. Andrei Abramian (Inst. for Problems in Mech. Eng. RAS, Bolshoy pr. V. O. 61, 199178 St. Petersburg, Russia, abramian@hidro.ipme.ru)

The phenomenon of wave localization in hydroelastic systems leads to the strength concentration of radiation fields. The linear method considers the process of localization to be the formation of nonpropagation waves (trapped modes phenomenon). The presence of such waves in the total wave packet points to the existance of mixed natural spectrum of differential operators describing the behavior of hydroelastic systems. The problem of liquid and oscillating structure interaction caused by the trapped modes phenomenon has been solved (membranes, dies, plates and different liquid models). The areas of wave localization have been determined. These areas depend on both the parameters of oscillating structures and the liquid waveguide parameters (the linear dimensions of the channel filled with liquid; the height from the bottom to the liquid surface; the liquid density gradient according to the height, etc.). Necessary and sufficient conditions for the low-frequency trapped modes and the highfrequency trapped modes have been found. The wave localization in nonlinear elastic structures interacting with liquid has been studied. Cases of weak and strong nonlinearity have been investigated. It has been shown that the slightly decreasing tail is added to nonlinear localized modes depending on the frequency of linear localized modes. The tail is caused by the interaction of the mode's continuous spectrum and the localized modes.

\section{$5: 20$}

3pUWb4. Trapping modes of oscillations in an infinitely long waveguide with submerged object in the form of a massive die. Dmitri Indeitsev (Inst. for Problems in Mech. Eng. RAS, Bolshoy pr. V.O. 61, St. Petersburg 199178, Russia, abramian@hidro.ipme.ru)

It is shown for an infinitely long waveguide-first in the form of a two-dimensional and then a three-dimensional channel-filled with an ideal compressible liquid with a free surface, that there can exist standing waves as well as traveling waves localized in the region of a dynamic submerged object-a massive die. The appearance of such waves is due to the presence of a mixed spectrum of characteristic frequencies of oscillation. In addition to the continuous spectrum, a point (discrete) spectrum distributed along the axis of the continuous spectrum is also present. A relation is found between the geometric parameters of the channel and the massive die for which this spectrum exists.
3pUWb5. Repulsion phenomena in the phase-velocity dispersion curves of circumferential waves on elastic cylindrical shells. Herbert Überall (Dept. of Phys., Catholic Univ., 6th and Michigan Ave. N.E., Washington, DC 20064), Gérard Maze, Fernand Léon, and Jean Ripoche (Univ. of Le Havre, 76610 Le Havre, France)

The complex eigenfrequencies of an evacuated, fluid-immersed infinite cylindrical shell, when plotted in the complex frequency plane, have been grouped into families corresponding to different types of circumferential waves. The real parts of these eigenfrequencies have been used to obtain dispersion curves versus frequency of the phase velocities of these circumferential waves, which are analogous to those of the Lamb waves on an elastic plate in vacuo labeled $A_{0}, A_{1}, \ldots$ and $S_{0}, S_{1}, \ldots$, but which contain an additional branch reminiscent of the fluid-borne Scholte-Stoneley wave. This branch forms together with the $A_{0}$-analog an interacting pair of dispersion curves which will be called $A_{0+}$ (upper branch) and $A_{0-}$ (lower branch). These exhibit a repulsion phenomenon near the ambient fluid sound speech analogous to that in perturbed, quasi-degenerate atomic levels. This is explained here by perturbation theory, showing that during repulsion the wave character gets exchanged so that at frequencies above the repulsion point $A_{0+}$ is a flexural and $A_{0-}$ a fluid-borne wave, and vice versa below the repulsion point.

\section{6:00}

3pUWb6. Localization in acoustic propagation in water with air cylinders. Zhen Ye and Emile Hoskinson (Dept. of Phys., Natl. Central Univ., Chungli, Taiwan, ROC)

Wave localization is the peculiar property of a scattering medium that completely blocks propagation, thus inducing a surprising phase transition, for example in optical or acoustic transparency or electrical conductivity. When localized, waves remain confined in space until dissipated. This property has been explained for randomly disordered scatterers. It is generally believed that disorder is an essential ingredient of localization, and in two dimensions (2D) waves are always localized with any amount of randomness. Here it is shown that waves are not always localized in 2D, but under proper conditions even periodic structures can achieve localization in the case of acoustic propagation through water containing an array of air cylinders. An essential component of localization in this case arises from the natural resonance and an amazing collective behavior of the cylinders. Exact numerical calculations reveal the unexpected result that localization is relatively independent of the precise location or organization of the scatterers. The results provide insight on 2D wave localization and offer an opportunity for further investigation of important questions about localization phenomena. [Work supported by NSC and NCU of ROC.] 